\title{
Astroglial Cx30 differentially impacts synaptic activity from hippocampal principal cells and interneurons
}

\author{
Eléonore Hardy ${ }^{1}$, Martine Cohen-Salmon², Nathalie Rouach ${ }^{1}$ \& Armelle Rancillac ${ }^{1 *}$
}

\begin{abstract}
${ }^{1}$ Neuroglial Interactions in Cerebral Physiopathology, Center for Interdisciplinary Research in Biology (CIRB), Collège de France, Centre National de la Recherche Scientifique (CNRS) Unité Mixte de Recherche UMR 7241, Institut National de la Santé et de la Recherche Médicale (INSERM) U1050, Labex Memolife, PSL-Research University, 75005 Paris, France.

${ }^{2}$ Physiology and Physiopathology of the Gliovascular Unit Research Group, Center for Interdisciplinary Research in Biology (CIRB), Collège de France, Centre National de la Recherche Scientifique (CNRS) Unité Mixte de Recherche UMR 7241, Institut National de la Santé et de la Recherche Médicale (INSERM) U1050, Labex Memolife, PSL-Research University, 75005 Paris, France.
\end{abstract}

Abbreviated title: Role of astroglial Cx30 on synaptic networks.

Keywords: neuroglial interactions, astrocytes, connexin 30, patch-clamp, synaptic transmission, excitation-inhibition balance, hippocampus.

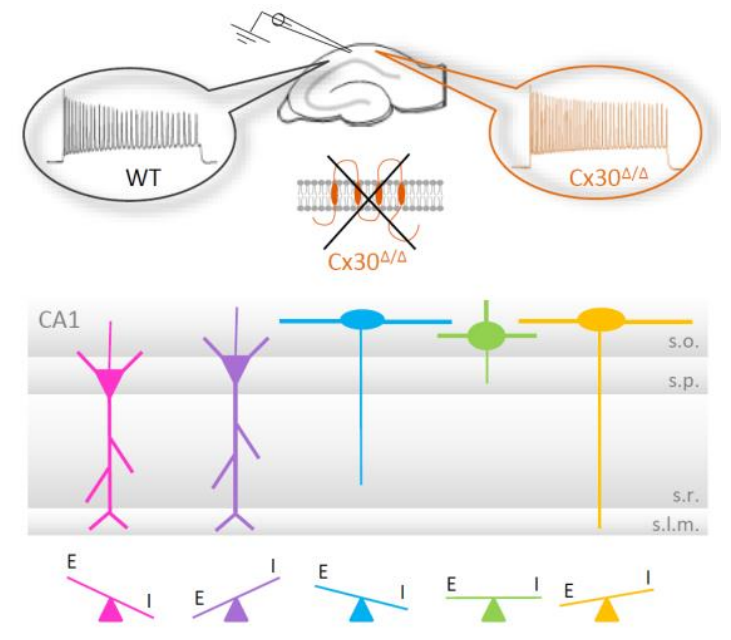

* Correspondence should be addressed to:

Dr. Armelle Rancillac

Neuroglial Interactions in Cerebral Physiopathology

Center for Interdisciplinary Research in Biology, CNRS UMR 7241, Inserm U1050

Collège de France

11 place Marcelin Berthelot 75005 Paris France

E-mail: armelle.rancillac@college-de-france.fr

Main points:

- Astroglial $\mathrm{C} \times 30$ alters the

electrophysiological properties of

hippocampal neuronal populations.

- The excitation-inhibition balance from

hippocampal neuronal subtypes is

distinctively regulated by astroglial $\mathrm{C} \times 30$. 


\section{Abstract}

Astrocytes play important roles in brain function via dynamic structural and functional interactions with neurons. Yet the underlying mechanisms remain poorly defined. A typical feature of astrocytes is the high expression of connexins, which mediate their extensive intercellular communication and regulate their structural properties. In particular, connexin $30(\mathrm{Cx} 30)$, one of the two connexins abundantly expressed by astrocytes, was recently shown to be a critical regulator of excitatory synaptic transmission by controlling the astroglial coverage of synapses. However, the role of Cx30 in the regulation of inhibitory synaptic transmission and excitatory/inhibitory balance remains elusive. Here, we investigated the role of astroglial $\mathrm{Cx} 30$ on the electrophysiological and morphological properties of five classes of hippocampal CA1 stratum oriens and pyramidale neurons, defined by the unsupervised Ward's clustering. Using $\mathrm{C} \times 30$ knockout mice, we found that $\mathrm{C} \times 30$ alters specific properties of some subsets of CA1 interneurons, such as resting membrane potential and sag ratio, while other parameters, such as action potential threshold and saturation frequency, were more frequently altered among the different classes of neurons. The excitationinhibition balance was also differentially and selectively modulated among the different neuron subtypes. Only slight morphological differences were observed on reconstructed neurons. Altogether, these data indicate that $\mathrm{Cx30}$ differentially alters the electrophysiological and morphological properties of hippocampal cell populations, and modulates both their excitatory and inhibitory inputs. Astrocytes, via Cx30, are thus active modulators of both excitatory and inhibitory synapses in the hippocampus. 


\section{Introduction}

In the mammalian brain, each neuron receives tens of thousands of excitatory $(E)$ and inhibitory (I) synaptic inputs. The ratio of excitatory to inhibitory input received by a neuron, termed Excitation-Inhibition (EI) balance, controls neuronal excitability, the spontaneous firing and/or its response to sensory inputs (Bhatia et al., 2019; Murphy \& Miller, 2009; Okun \& Lampl, 2008; Wehr \& Zador, 2003) and is thought to be critical for information processing in neural circuits. At the cellular level, the density of excitatory and inhibitory synapses seems to be precisely balanced across the various dendrites of single neurons to strongly impact its firing output (lascone et al., 2020). Therefore, disruption of the El balance is linked to epilepsy and numerous psychiatric diseases, including schizophrenia and autism spectrum disorders (Ferguson \& Gao, 2018; Lee et al., 2017; Nelson \& Valakh, 2015; Yizhar et al., 2011).

An appropriate E/l ratio is regulated by different cell types. In particular, GABAergic interneurons control the spike generation of neighbouring pyramidal neurons (Roux and Buzsáki 2015), allows a feed-forward control of neuronal networks for precise temporal signaling (Kanichay and Silver 2008) and contributes to the generation of synchronized oscillations in brain networks (Adesnik 2018). Astrocytes, a major glial cell population are also key elements involved in synapse maturation, remodeling and transmission (Araque et al., 2014; Eroglu \& Barres, 2010; Rusakov, 2015). The close vicinity of astrocytes to synapses, the so-called the tripartite synapse, allows astrocytes to sense neuronal activity by their ion channels, neurotransmitter receptors and transporters. Astrocytes are strategically located to allow fine regulations of synaptic activity. By their ability to uptake or release neuroactive factors, they can modulate neighboring pre- and postsynaptic elements (Covelo \& Araque, 2018; Genoud et al., 2006; Oliet et al., 2001). Whereas neurons excitation relies on membrane voltage 
changes, astrocytes activation results in intracellular calcium increase. Another important feature of astrocytes is their high expression levels of the gap junction protein connexins (Cx) 30 and 43, also regulating brain homeostasis through their role in potassium buffering, intercellular communication and nutrients transport (U. Pannasch et al., 2011; Ulrike Pannasch et al., 2014). However, Cx are not systematically forming a pore to other astrocytes and can also exchange with the extracellular space when forming hemichannels. Recent studies also reported channel-independent functions of Cx involving intracellular signaling, protein interactions and cell adhesion (Ghézali et al., 2016; Ulrike Pannasch et al., 2014). Indeed, Cx30 expression was shown to regulate the insertion of astroglial processes into synaptic clefts, controlling the efficacy of glutamate clearance and thereby the synaptic strength and long-term plasticity of excitatory terminals.

Beyond the regulation of excitatory synapses, Cx30 may also modulate neuronal network activities by multiple mechanisms such as modulation of astroglial coverage of inhibitory synapses, release of neuroactive factors via hemichannels, ion and neurotransmitter extracellular homeostasis as well as metabolic support via gap junctions. Indeed, beyond its role in ion, neurotransmitter or energy metabolite trafficking via gap junction channels (Hansen et al., 2014; Rouach et al., 2008), Cx30 has also recently been shown to form hemichannels in hippocampal astrocytes (Ghézali et al., 2019). Further, since astrocytes express GABA transporter 1 (GAT1) and 2 (GAT2) (Ghirardini et al., 2018), the vicinity of astroglial processes to inhibitory synapses could also directly regulate GABA clearance in the hippocampus (Juhász et al., 1997; Richards \& Bowery, 1996).

The aim of the study was thus to determine the functional impact of astroglial Cx30 on the morphological and electrophysiological properties of hippocampal CA1 neuronal 
populations. The hippocampus contains a large diversity of local-circuit inhibitory interneurons endowed with different electrophysiological features, morphological shapes and molecular markers (Freund \& Buzsáki, 1996; Klausberger \& Somogyi, 2008; Pelkey et al., 2017). Importantly, interneurons play highly specific roles and are crucial elements to control information processing performed by pyramidal cells, from input integration to output generation and synchronization of network activities. To restrain this diversity, we here focused our study on neurons located in stratum oriens and the pyramidal layer of the hippocampal CA1 region, where we combined patchclamp recordings and morphological reconstructions of different neuronal cell types in wildtype and knockout mice for Cx30. 


\section{Materials and Methods}

Animals. C57BL/6J wildtype (WT) and $\mathrm{C} \times 30^{\Delta / \Delta}$ mice (transgenic knock-out animal deficient for Cx30 expression (Boulay et al., 2013)) were used for all experiments. $\mathrm{C} \times 30^{\Delta / \Delta}$ mice are indeed a more appropriate model to study the impact of $\mathrm{C} \times 30$ deletion than $\mathrm{C} \times 30^{-/-}$mice, where the removal of the $\mathrm{C} \times 30$ exon and its replacement by a lacZ reporter gene and a neo resistance cassette have been suggested to strongly impact the expression of Cx26 in the inner ear and contributes to deafness (Boulay et al., 2013). Instead, more than half of Cx26 expression is preserved in the inner ear of Cx30 ${ }^{\Delta / \Delta}$ mice, which are not deaf (Boulay et al., 2013). Further, we used C57BL/6J wildtype mice for control conditions as $\mathrm{Cx} 30 \mathrm{fl} / \mathrm{fl}$ adult mice show a reduction by almost $40 \%$ in $\mathrm{C} \times 30$ expression. Mice were housed in a temperature-controlled $\left(20-22^{\circ} \mathrm{C}\right)$ room under a 12-hour light-dark cycle with ad libitum access to food and water. All animal procedures were conducted in strict compliance with our institutional protocols and were approved by the European Community Council Directive of 1 January 2013 (2010/63/UE) and the local ethics committee (Comité d'éthique en matière d'expérimentation animal number 59, C2EA -59, 'Paris Centre et Sud'). Accordingly, all mice were backcrossed to the C57BL6 background for ten generations. Mice of both sexes were used at postnatal days (P19-23).

Preparation of acute hippocampal slices. Animals were decapitated and their brains were quickly extracted and submerged in cold slicing artificial cerebrospinal fluid (aCSF, $4{ }^{\circ} \mathrm{C}$ ) containing (in $\mathrm{mM}$ ): $119 \mathrm{NaCl} ; 2.5 \mathrm{KCl} ; 2.5 \mathrm{CaCl} 2 ; 26.2 \mathrm{NaHCO}$; $1 \mathrm{NaH} 2 \mathrm{PO} 4 ; 1.3 \mathrm{MgSO} 4 ; 11 \mathrm{D}$-glucose $(\mathrm{pH}=7.35)$. Brains were constantly oxygenated with $95 \% \mathrm{O}_{2}-5 \% \mathrm{CO}_{2}$. Hippocampal brain slices (400 $\mu \mathrm{m}$ thick) were cut with a vibratome (VT1200S; Leica) and transferred to a constantly oxygenated (95\% 
$\mathrm{O}_{2}-5 \%$ CO2) holding chamber containing aCSF. Subsequently, individual slices were placed in a submerged recording chamber maintained at $32{ }^{\circ} \mathrm{C}$ and perfused with oxygenated aCSF, and placed under an upright microscope (AxioScop, Zeiss) equipped with a CCD camera (C 2450, Hamamatsu) for observation.

Drugs. Tetrodotoxin (TTX) (1 $\mu$ M; Sigma) and biocytin (Sigma) were used in this study.

Whole-cell patch-clamp recordings. Slices were maintained immersed and continuously surperfused at $1-2 \mathrm{~mL} / \mathrm{min}$ with oxygenated aCSF. Electrophysiological experiments were performed with a MultiClamp700B (Axon Instruments) amplifier connected to an acquisition board (Digidata 1440; Axon Instruments) attached to a computer running the pCLAMP software (Axon Instruments). The targeted neurons were located in the stratum oriens and pyramidale of CA1.

Recordings in the whole-cell patch-clamp configuration were performed with patchclamp pipettes (3-6 M 2 ) filled with an internal solution containing (in $\mathrm{mM}$ ): $129 \mathrm{~K}$ gluconate; 10 EGTA; 10 HEPES and 2 ATP-Mg (pH 7.2); 285-295 mOsm; 2 mg/mL biocytin (Sigma Aldrich) were added to this internal solution to allow morphological analysis. The liquid junction potential of the patch pipette and the perfused extracellular solution was $11 \mathrm{mV}$ and was not applied to the data. The pipette was slowly brought to the selected neuron for recording in whole-cell configuration using infrared videomicroscopy guidance and clamped at $-60 \mathrm{mV}$. The intrinsic neuronal membrane properties were assessed in current-clamp by applying current steps (800 ms) from $100 \mathrm{pA}$ until firing saturation, in $10 \mathrm{pA}$ increments.

For each neuron, 28 electrophysiological parameters were measured according to the Petilla terminology (Ascoli et al., 2008), as fully described in (Karagiannis et al., 2009). 
Briefly, for the parameter 1 (p1), the resting membrane potential was measured immediately after passing to whole-cell configuration. (p2) Input resistance $(\mathrm{Rm})$ and (p3) the membrane time constant ( $\mathrm{Tm}$ ) was determined at the beginning of the voltage response of a hyperpolarization current step (-10 pA, $800 \mathrm{~ms})$. The time constant was determined by fitting this voltage response to a single exponential. (p4) The membrane capacitance $(\mathrm{Cm})$ was calculated according to the equation $\mathrm{Cm}=\mathrm{Tm} / \mathrm{Rm}$. Under our conditions, injection of hyperpolarizing current pulses often induced a hyperpolarization-activated cationic current (Ih) that followed the initial hyperpolarization peak, known as a sag. (p5) Rhyp was measured as the slope of the linear portion of an I-V plot, measured at the beginning $(0-0.1 \mathrm{~s} ;-100$ to $0 \mathrm{pA})$. (p6) $R_{\text {sag }}$ was measured as the slope of the linear portion of a current-voltage (IV) plot, measured at the end of the hyperpolarizing current pulses (0.7-0.8 s; -100 to $0 \mathrm{pA})$. (p7) $\Delta \mathrm{R}_{\text {sag }}$ corresponds to $\left(\mathrm{R}_{\mathrm{sag}}-\mathrm{R}_{\text {hyp }}\right) / \mathrm{R}_{\text {sag. }}$ (p8) Action potential threshold corresponded to the voltage threshold of the first action potential elicited by a current ramp. (p9) The rheobase was the minimum current that elicited an action potential. (p10) The first spike latency was computed as the time needed to elicit a spike after the onset of a current pulse corresponding to the rheobase. Neurons have been described to exhibit a wide range of firing behaviors around threshold, some exhibiting bursting, adapting, regular or irregular trains of action potentials. In order to describe this variety of behaviors with quantitative parameters, the interspaced intervals measured in response to the minimal current injection eliciting more than three action potentials were plotted and fitted to a linear curve. (p11) Adaptation (mthreshold) was computed as the slope of the linear fit and (p12) the minimal steady state frequency ( $F_{\text {threshold }}$. (p13 and p15) Amplitudes of the first (A1) and second (A2) action potential (AP) were measured from the threshold to the positive peak on the first step, where at 
least 3 APs were induced. (p14 and p16) AP durations were measured at halfamplitude (D1 and D2). (p17) The amplitude and (p18) duration of the maximal afterhyperpolarization (AHP) was measured for the first AP. (p19) The amplitude and (p20) duration of the maximal after-depolarization (ADP) was measured for the first AP. (p21) Amplitude variation (Var A) and (p22) duration variation (Var D) were computed as (A1-A2)/A1*1000 and (D2-D1)/D1*1000 respectively. At higher stimulation intensities, the maximal firing rate was defined as the last trace before prominent reduction of the action potential amplitude, indicative of a saturated discharge. (p23) Imax, is the minimal current inducing saturating frequencies. To take into account the biphasic spike frequency adaptation (early and late), instantaneous firing frequency was fitted to a single exponential with a sloping baseline, according to the equation $F_{\text {sat }}=A_{\text {sat }} \times E^{-t / \text { sat }}+t \times m_{\text {sat }}+F_{\max }$, where (p24) $A_{\text {sat }}$ corresponds to the amplitude of early frequency adaptation, (p25) Tsat to the time constant of early adaptation, (p26) $F_{\max }$ the maximal steady-state frequency and $(\mathbf{p} 27) \mathrm{m}_{\text {sat }}$ to the slope of late adaptation. During the $800 \mathrm{~ms}$ depolarization protocol, a pronounced reduction of the amplitude of the action potentials was followed by an increase of the spike amplitude. This difference of action potential amplitude was termed the amplitude accommodative hump (p28).

Data were rejected if the access resistance increased over $25 \mathrm{MOhm}$ during a recording or changed by more than $25 \%$ during the recording. Miniature currents were recorded in voltage-clamp mode at a holding potential of $-60 \mathrm{mV}$, which was less negative than the reversal potential for sIPSC. Indeed, the pipette solution contained a low concentration of $\mathrm{Cl}(1 \mathrm{mM})$, resulting in an $\mathrm{E}_{\mathrm{Cl}}$ of $-111 \mathrm{mV}$. Therefore, inward currents were considered as SEPSCs and outward currents as SIPSCs (Sangare et al., 2016). Miniature events were recorded by including TTX (1 mM) in the aCSF. Data 
were filtered at $2 \mathrm{kHz}$, digitized at $10 \mathrm{kHz}$ and acquired on-line using the PCLAMP 9 (Clampex) software (Axon Instruments). The recorded currents were subsequently analyzed using Clampfit.

Ward's Clustering and Silhouette. Unsupervised clustering was performed essentially as described previously (Karagiannis et al., 2009; Perrenoud et al., 2012). Briefly, from the 28 electrophysiological parameters we only kept 13 electrophysiological parameters to avoid a bias of redundancy. These 13 electrophysiological parameters: $R_{m}(p 2), T_{m}(p 3), R_{h y p}(p 5), R_{s a g}(p 6), \Delta R_{\text {sag }}(p 7), A 1$ (p13), D1 (p14), AHP amplitude (p17), AHP duration (p18), Asat (p24), Tsat (p25), $F_{\max }$ (p26), msat (p27), were standardized by centering and reducing all of the values of the 62 neurons in WT mice and 44 in $C \times 30^{\Delta / \Delta}$ mice, using Statistica 6 software (Statsoft, Tulsa, OK, USA). We built the scree plot of eigenvalues and PCA component (supplementary Fig. S1). We selected the first five components with an Eigenvalue $\geq 1$, and which explain almost $80 \%$ of variance. We then conducted a cluster analysis using unsupervised clustering performed by Ward's method (Ward, 1963) that does not require to set a number of classes to be characterized. The clusters generated by Ward's method were then corrected using the K-means algorithm by using Matlab software (MathWorks). To quantitatively assess the quality of a clustering, silhouette analysis of the K-means clustering was performed. A positive silhouette value indicates that on average, the neuron is closer to the neurons of its own cluster than from the neurons belonging to other clusters in the parameter space. On the opposite, a negative value indicates a potential misclassification. 
Immunohistochemestry. After biocytin injection into the recorded neurons, slices were fixed in 4\% PFA overnight and washed 3 times in PBS next day. For immunohistochemistry, slices were blocked with PBS-Gelatin-Triton (PBS with 0.2\% of gelatin and $1 \%$ of Triton), for $1 \mathrm{~h}$ at room temperature, incubated $2 \mathrm{~h}$ with Streptavidin conjugate to alexa fluor 555 (Thermofischer S21381 at 1:200) in the blocking solution and washed three times with PBS before mounting with Fluoromount (Invitrogen).

Morphological analysis. In order to describe variations of morphology between recorded neurons, 22 parameters related to features of the soma and dendrites were extracted by using Neurolucida explorer (MBF Bioscience). For morphologies of somata, (p29) the soma area, (p30) perimeter, (p31) form factor, and (p32) maximal, (p33) minimal diameter passing through the centroid (Feret) was computed. The degree of flatness of a contour shape, taken as the ratio of its minimum diameter to its maximum diameter, was indicated as (p34) the aspect ratio. (p35) The somatic solidity, the ratio of the somata area as a whole over the convex area, where values closer to 1 represent more solid (i.e. smooth, uniform) somata was calculated for each soma. (p36) Convexity $=($ Convex Perimeter $) /($ Perimeter $)$. This parameter is indicative of the somatic morphological profile. The measurement of how closely this shape approached that of a circle was assessed throughout (p37) the roundness. Finally (p38) somatic compactness $=[\sqrt{ }(4 / \pi) \times$ area $] /($ feret max $)$; numerical values of somatic compactness closer to 1 represent a more compact soma.

In order to be able to describe the differences of dendritic arborizations, we systematically quantified the following parameters: (p39) Number of primary dendrites, (p40) total dendritic length, (p41) total dendritic surface, (p42) total dendritic volume, 
(p43) ratio of the total dendritic length on the total dendritic surface, (p44) highest dendritic order segment, (p45) average tortuosity of dendritic segments (defined as the ratio of the length of the segment over the straight line path between its extremities) and (p46) number of dendritic nodes. We performed a Sholl analysis (Sholl 1953) to describe differences between neuronal arborization. (p47) The length of the dendritic arborization that could be enclosed within a $100 \mu \mathrm{m}$ radius circle around the cell body, (p48) between $100 \mu \mathrm{m}$ and $200 \mu \mathrm{m}$ radii, (p49) between $200 \mu \mathrm{m}$ and $300 \mu \mathrm{m}$ radii, and (p50) outside a $300 \mu \mathrm{m}$ radius was systematically extracted. The analysis of neuronal morphologies served a posteriori to validate the correspondence of clusters, as well as their typical electrophysiological features.

Statistics. All data are expressed as the mean \pm standard error of the mean (SEM). Statistical significance was determined using a Kruskal-Wallis test and Dunn's multiple comparisons test for testing the difference between clusters. Mann-Whitney $U$-test for testing the difference in two population values and using a Kolmogorov-Smirnov test for testing the difference in two-population probability distribution. $P$-values of $\leq 0.05$ were considered statistically significant. In all cases, $\mathrm{n}$ refers to the number of examined neurons. Asterisks indicate statistical significance $\left({ }^{*} P<0.05,{ }^{* *} P<0.01,{ }^{* * *} P<0.001\right)$. 


\section{RESULTS}

To decipher how $\mathrm{C} \times 30$ expression regulates neuronal information processing, we investigated the impact of $\mathrm{C} \times 30$ deletion on the neuronal electrophysiological and morphological properties in the CA1 region of the hippocampus using wildtype and Cx30 deficient mice. As Cx30 expression might differentially alter specific subset of neurons, we first used the electrophysiological properties of the recorded neurons to perform an unsupervised classification of CA1 neurons from wildtype mice. Then, we performed similar experiments in $\mathrm{C} \times 30$ deficient mice and compared the electrophysiological and morphological properties of each neuronal cluster to those of wildtype mice.

\section{Unsupervised classification of CA1 neurons from wildtype mice}

Sixty-two neurons were characterized by patch-clamp recordings within the hippocampal CA1 stratum oriens and pyramidal cell layer. Twenty-eight electrophysiological features were measured for each cell from responses to hyperpolarizing and depolarizing current pulses in whole-cell current-clamp mode. Five principal components were selected from a principal component analysis performed on the thirteen discriminative electrophysiological parameters (see Materials and Methods). Based on these parameters, we applied the unsupervised clustering Ward's method (Ward, 1963) to avoid personal bias of determination and to group cells with large similarities into distinct subpopulations. A clustering tree was built, representing individual cells from its leaves and grouping them into the branched ramification up to a common root (Fig. 1a). 


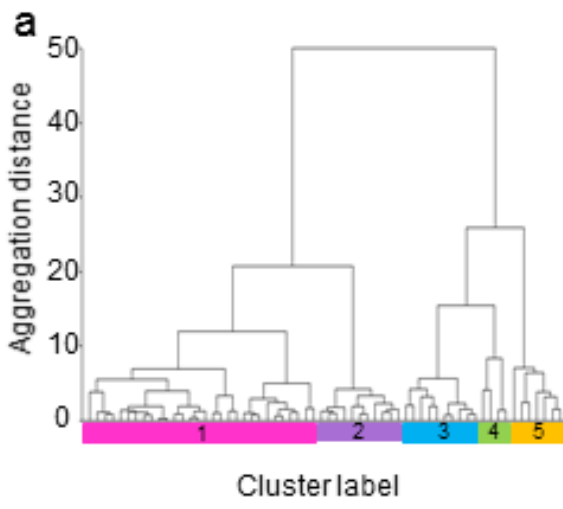

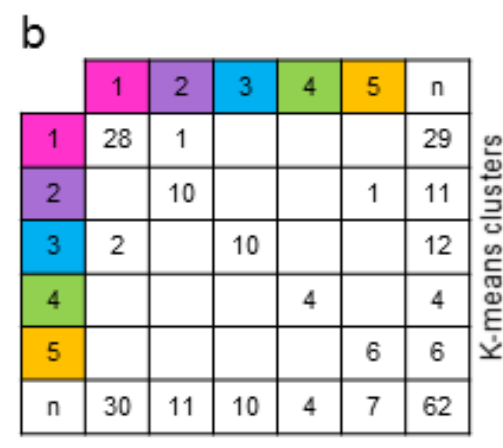

Ward clusters
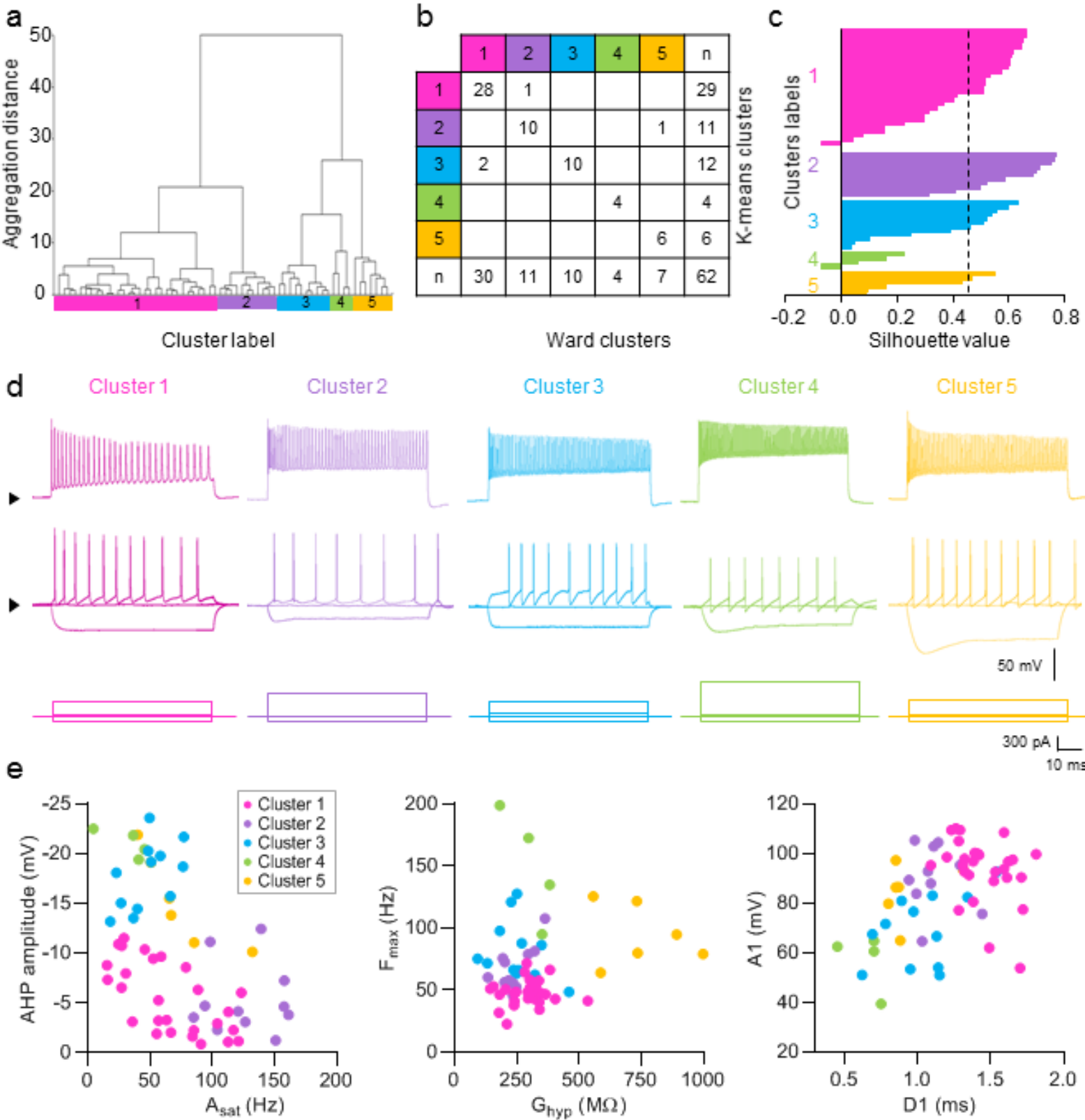

Cluster 3

Cluster 4

Cluster 5

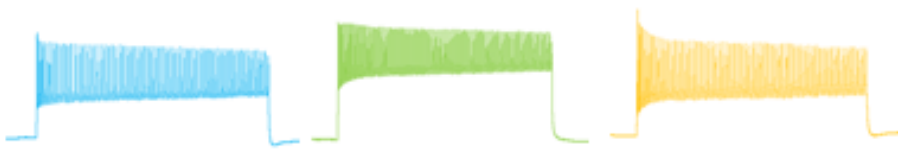

$50 \mathrm{mv}$
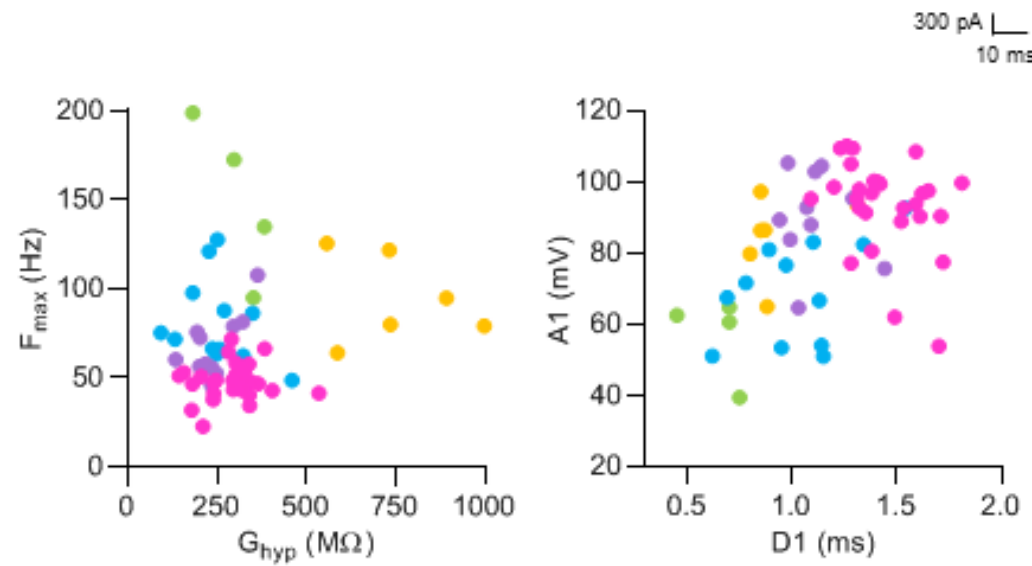

Figure. 1. Unsupervised clustering of hippocampal CA1 neurons based on their electrophysiological properties recorded in wildtype mice. (a) Ward's clustering of 62 CA1 neurons. Individual cells are represented along the $x$-axis. The $y$-axis represents the average within-cluster linkage distance in a space of 5 principal component extracted from 13 electrophysiological variables. Five clusters, 1 (in pink), 2 (in violet), 3 (in blue), 4 (in green) and 5 (in yellow) were identified. (b) Clusters generated by Ward's method in (A) were corrected using the clustering output generated by the K-means algorithm. (c) The silhouette analysis was used to assess the quality of the clustering that was optimal for 5 clusters with a mean value of 0.418 (dash line). (d) Typical electrophysiological behavior of neurons from each cluster recorded in current-clamp. Neurons from cluster 1 (pink), 2 (violet), 3 (blue), 4 (green) and 5 (yellow) were recorded using whole-cell patch-clamp recordings in response to the application of current pulses (bottom traces). Strong depolarizing current evoked a 
high and sustained firing in all clusters (top traces). Typical voltage responses to hyperpolarizing current step injection $(-100 \mathrm{pA})$, at resting membrane potential $(0 \mathrm{pA})$ and when depolarized at their spiking threshold evoked distinct responses in all clusters (middle traces). (e) Individual values for each cell among cluster for AHP amplitude and the amplitude of adaptation. Individual values for each cell among cluster for frequency and Rhyp. Individual values for each cell among cluster for amplitude and duration of the first action potential.

The reliability of Ward's clustering was corrected using the K-mean algorithm. Two cells of cluster 1 were reassigned to cluster 3 , one cell of cluster 2 was reassigned to cluster 1 , and 1 cell from the cluster 5 was reassigned to cluster 2 (Fig. 1b). In order to determine whether the allocation of neurons into five groups was the optimal outcome of the clustering process, our results were compared with clustering trials whereby thresholds were set to define fewer or higher numbers of groups. We established that a cluster analysis yielding five groups was the best outcome with a mean silhouette value of 0.418 (Fig. 1c). Moreover, neurons from these clusters exhibited district electrophysiological behavior (Fig. 1d). Thus, the subdivision of CA1 neurons into five main groups was retained for further analysis.

\section{Electrophysiological Diversity of the Hippocampal CA1 Stratum Oriens and Stratum Pyramidal Neurons}

To characterize the neuronal diversity of CA1 stratum oriens and pyramidale neurons, the 28 discriminative electrophysiological features measured on our 62 cells were compared among the five identified clusters (Table 1). Electrophysiological data regarding subthreshold properties showed in particular that cluster 1 neurons displayed a significantly higher membrane capacitance $(\mathrm{p} 4)$ compared to other groups 
(Fig. 1e; Table 1). Neurons from cluster 5 were also characterized by an important Ih current, and a higher membrane time constant (p3), slope (p5, p6) and a smaller sag ratio (p7) compared to other groups (Table 1).

Table 1. Comparison of passive membrane properties among CA1 neuron subtypes in wildtype mice.

\begin{tabular}{|c|c|c|c|c|c|}
\hline & $\begin{array}{l}\text { Cluster } 1 \\
(n=29)\end{array}$ & $\begin{array}{c}\text { Cluster } 2 \\
(n=11)\end{array}$ & $\begin{array}{c}\text { Cluster } 3 \\
(\mathrm{n}=12)\end{array}$ & $\begin{array}{c}\text { Cluster } 4 \\
(n=4)\end{array}$ & $\begin{array}{c}\text { Cluster } 5 \\
(n=6)\end{array}$ \\
\hline (p1) RMP (mV) & $\begin{array}{c}-57.8 \pm 1.5 \\
-\end{array}$ & $\begin{array}{c}-57.8 \pm 1.4 \\
-\end{array}$ & $\begin{array}{c}-52.5 \pm 1.4 \\
-\end{array}$ & $\begin{array}{c}-55.3 \pm 4.9 \\
-\end{array}$ & $\begin{array}{c}-58.7 \pm 1.9 \\
-\end{array}$ \\
\hline (p2) $\mathbf{R}_{\mathbf{m}}(\mathrm{M} \Omega)$ & $\begin{array}{c}342.9 \pm 19.9 \\
-\end{array}$ & $\begin{array}{c}320.7 \pm 28.4 \\
-\end{array}$ & $\begin{array}{c}338.5 \pm 50.9 \\
-\end{array}$ & $\begin{array}{c}415.9 \pm 74.3 \\
-\end{array}$ & $\begin{array}{c}802.1 \pm 142.4 \\
-\end{array}$ \\
\hline (p3) $\mathbf{T}_{\mathbf{m}}(\mathrm{ms})$ & $\begin{array}{c}30.1 \pm 1.7 \\
{ }^{*} 2,{ }^{* *} 3\end{array}$ & $\begin{array}{c}19.6 \pm 1.7 \\
{ }^{*} 1,{ }^{* *} 5\end{array}$ & $\begin{array}{l}17.7 \pm 2.6 \\
\star \star 1 \\
{ }^{* * *} 5\end{array}$ & $\begin{array}{c}25.3 \pm 5.6 \\
-\end{array}$ & $\begin{array}{c}44.5 \pm 5.9 \\
\star \star 2,{ }^{* \star *} 3\end{array}$ \\
\hline (p4) $C_{m}(p F)$ & $\begin{array}{c}88.8 \pm 3.5 \\
* \star * 2,5,3\end{array}$ & $\underset{* \star * \star 1}{62.8 \pm 4.4}$ & $\underset{* * * 1}{53.2 \pm 5.4}$ & $\begin{array}{c}59.7 \pm 3.9 \\
-\end{array}$ & $\underset{* \star * \star * 1}{43.1 \pm 6.1}$ \\
\hline (p5) $\boldsymbol{R}_{\text {hyp }}(\mathrm{M} \Omega$ ) & $\begin{array}{c}292.3 \pm 15.2 \\
* * 5\end{array}$ & $\begin{array}{c}241.2 \pm 19.5 \\
\star \star \star \\
\end{array}$ & $\underset{* \star 5}{253.2 \pm 28.0}$ & $\begin{array}{c}304.0 \pm 44.1 \\
-\end{array}$ & $\begin{array}{c}750.9 \pm 69.9 \\
{ }^{\star \star} 1,3,{ }^{\star \star \star} 2\end{array}$ \\
\hline (p6) $\mathbf{R}_{\text {sag }}(M \Omega)$ & $\begin{array}{c}242.7 \pm 12.9 \\
* 5\end{array}$ & $185.0 \pm 14.8$ & $\begin{array}{c}237.9 \pm 27.8 \\
* 5\end{array}$ & $\begin{array}{c}239.6 \pm 28.4 \\
-\end{array}$ & $\begin{array}{c}569.2 \pm 80.4 \\
{ }^{\star \star} 1,3,{ }^{\star \star \star} 2\end{array}$ \\
\hline (p7) $\Delta \mathbf{R}_{\text {sag }}(\%)$ & $\begin{array}{c}83.3 \pm 1.2 \\
-\end{array}$ & $\begin{array}{c}77.2 \pm 1.8 \\
* \star 3\end{array}$ & $\begin{array}{c}93.3 \pm 2.8 \\
* \star 5,2\end{array}$ & $\begin{array}{c}80.2 \pm 4.8 \\
-\end{array}$ & $\begin{array}{c}75.5 \pm 6.5 \\
{ }^{* * 3}\end{array}$ \\
\hline
\end{tabular}

$n$, Number of cells; Kruskal-Wallis and Dunn's multiple comparisons test. (pNumber) refer to the electrophysiological parameters described in the material et methods section. Asterisks indicate statistical significance $\left({ }^{*} P<0.05,{ }^{* *} P<0.01,{ }^{* *} P<0.001\right)$ of the cluster in the column relative to the other clusters represented by their corresponding number.

When depolarized just above threshold, neurons from cluster 4 significantly exhibited a higher rheobase (p9) than neurons from cluster 1 and 5 (Table 2). Neurons from cluster 5 displayed significant shorter first spike latency ( $p 10)$, compared to neurons from cluster 1 and 3 . No significant differences were observed regarding adaptation (p11) and steady state firing frequency (p12) at threshold currents between clusters (Table 2). 
Table 2. Comparison of just above threshold properties among CA1 neuron subtypes in wildtype mice

\begin{tabular}{|c|c|c|c|c|c|}
\hline & $\begin{array}{c}\text { Cluster } \mathbf{1} \\
(\mathrm{n}=29)\end{array}$ & $\begin{array}{c}\text { Cluster } 2 \\
(\mathrm{n}=11)\end{array}$ & $\begin{array}{c}\text { Cluster } 3 \\
(n=12)\end{array}$ & $\begin{array}{c}\text { Cluster } 4 \\
(n=4)\end{array}$ & $\begin{array}{c}\text { Cluster } 5 \\
(\mathrm{n}=6)\end{array}$ \\
\hline (p8) $\mathbf{A} \boldsymbol{P}_{\text {th }}(\mathrm{mV})$ & $\begin{array}{c}-51.5 \pm 0.8 \\
* 3\end{array}$ & $\begin{array}{c}-52.6 \pm 1.6 \\
-\end{array}$ & $\begin{array}{c}-44.9 \pm 1.8 \\
* 1\end{array}$ & $\begin{array}{c}-42.6 \pm 3.7 \\
-\end{array}$ & $\begin{array}{c}-50.6 \pm 2.5 \\
-\end{array}$ \\
\hline (p9) Rheobase (pA) & $\begin{array}{c}17.2 \pm 1.9 \\
* 4\end{array}$ & $\begin{array}{c}19.1 \pm 3.1 \\
-\end{array}$ & $\begin{array}{c}37.5 \pm 11.2 \\
-\end{array}$ & $\begin{array}{c}45.0 \pm 9.6 \\
* 1,5\end{array}$ & $\begin{array}{c}11.7 \pm 1.7 \\
* 4\end{array}$ \\
\hline (p10) $\mathbf{1}^{\text {st }}$ spike (ms) & $\begin{array}{c}192.2 \pm 36.5 \\
* 5\end{array}$ & $\begin{array}{c}110.5 \pm 36.5 \\
-\end{array}$ & $\underset{* \star 5}{252.9 \pm 54.8}$ & $\begin{array}{c}112.2 \pm 35.9 \\
-\end{array}$ & $\begin{array}{c}25.9 \pm 3.8 \\
{ }^{*} 1,{ }^{\star \star} 3\end{array}$ \\
\hline$(\mathrm{p} 11) \mathbf{m}_{\mathrm{th}}(\mathrm{Hz} / \mathrm{s})$ & $\begin{array}{c}-8.9 \pm 2.5 \\
-\end{array}$ & $\begin{array}{c}-9.6 \pm 6.9 \\
-\end{array}$ & $\begin{array}{c}-6.8 \pm 3.3 \\
-\end{array}$ & $\begin{array}{c}-15.7 \pm 10.9 \\
-\end{array}$ & $\begin{array}{c}-2.2 \pm 1.8 \\
-\end{array}$ \\
\hline$(\mathrm{p} 12) \mathbf{F}_{\text {th }}(\mathrm{Hz})$ & $\begin{array}{c}12.2 \pm 1.5 \\
-\end{array}$ & $\begin{array}{c}11.6 \pm 3.8 \\
-\end{array}$ & $\begin{array}{c}14.3 \pm 4.0 \\
-\end{array}$ & $\begin{array}{c}25.1 \pm 17.6 \\
-\end{array}$ & $\begin{array}{c}15.6 \pm 5.3 \\
-\end{array}$ \\
\hline
\end{tabular}

$n$, Number of cells; Kruskal-Wallis and Dunn's multiple comparisons test. (pNumber) refer to the electrophysiological parameters described in the material et methods section. Asterisks indicate statistical significance $\left({ }^{*} P<0.05,{ }^{* *} P<0.01\right)$ of the cluster in the column relative to the other clusters represented by their corresponding number.

Comparison of action potential properties among CA1 neurons from stratum oriens and pyramidal essentially revealed that cluster 1 neurons displayed a regular-spiking activity characteristic of pyramidal cells (Staff et al., 2000). These neurons were essentially characterized by a long action potential duration (p14 and p16; Fig. 1e), whereas neurons from cluster 2 are essentially characterized by a longer AHP ( $p 18)$. In contrast to neurons from the other clusters, neurons from cluster 1 and 2 displayed less pronounced AHP (p17; Fig. 1e). Neurons from cluster 4 are essentially characterized by their high firing frequency at saturating (p26; Table 3), a typical feature of fast-spiking interneurons. 
Table 3. Comparison of action potential properties among CA1 neurons in wildtype mice

\begin{tabular}{|c|c|c|c|c|c|}
\hline & $\begin{array}{c}\text { Cluster } 1 \\
(n=29)\end{array}$ & $\begin{array}{c}\text { Cluster } 2 \\
(n=11)\end{array}$ & $\begin{array}{c}\text { Cluster } 3 \\
(n=12)\end{array}$ & $\begin{array}{c}\text { Cluster } 4 \\
(n=4)\end{array}$ & $\begin{array}{c}\text { Cluster } 5 \\
(n=6)\end{array}$ \\
\hline (p13) A1 (mV) & $\begin{array}{c}92.6 \pm 2.5 \\
{ }^{* *} 4,{ }^{* * *} 3\end{array}$ & $\begin{array}{c}95.7 \pm 6.6 \\
* 3,4\end{array}$ & $\begin{array}{c}69.3 \pm 4.1 \\
* * 2,{ }^{* * *} 1\end{array}$ & $\begin{array}{c}56.8 \pm 5.9 \\
* 2,{ }^{* *} 1\end{array}$ & $\begin{array}{c}84.8 \pm 4.7 \\
-\end{array}$ \\
\hline (p14) D1 (ms) & $\begin{array}{c}1.5 \pm 0.0 \\
\star \star 2,5,{ }^{* \star *} 3,4\end{array}$ & $\begin{array}{c}1.1 \pm 0.1 \\
\star \star 1\end{array}$ & $\begin{array}{c}1.0 \pm 0.1 \\
* \star * \\
\text { ** }\end{array}$ & $\begin{array}{c}0.7 \pm 0.1 \\
* \star * 1\end{array}$ & $\begin{array}{c}0.9 \pm 0.1 \\
* * 1\end{array}$ \\
\hline (p15) A2 (mV) & $\begin{array}{c}91.2 \pm 2.4 \\
* \star \star 3,4\end{array}$ & $\begin{array}{c}94.8 \pm 6.3 \\
\quad * * 3,4\end{array}$ & $\begin{array}{c}69.1 \pm 3.9 \\
* 2,{ }^{* *} 1\end{array}$ & $\begin{array}{c}56.9 \pm 5.8 \\
* 2,{ }^{\star *} 1\end{array}$ & $\begin{array}{c}84.8 \pm 4.7 \\
-\end{array}$ \\
\hline (p16) D2 (ms) & $\begin{array}{l}1.5 \pm 0.0 \\
{ }^{\star} 2,{ }^{* * *} 3,4\end{array}$ & $\begin{array}{c}1.1 \pm 0.1 \\
* 1\end{array}$ & $\begin{array}{c}1.0 \pm 0.1 \\
* * * 1\end{array}$ & $\begin{array}{c}0.7 \pm 0.1 \\
* \star * \\
0\end{array}$ & $\begin{array}{c}0.9 \pm 0.1 \\
* * 1\end{array}$ \\
\hline (p17) AHP (mV) & $\begin{array}{c}-5.1 \pm 0.7 \\
{ }^{*} 5,{ }^{* * *} 3,4\end{array}$ & $\begin{array}{c}-5.3 \pm 1.1 \\
{ }^{* *} 3,4\end{array}$ & $\begin{array}{c}-17.8 \pm 0.9 \\
{ }^{* *} 2,{ }^{* *} 1\end{array}$ & $\begin{array}{c}-21.1 \pm 0.7 \\
{ }^{* *} 2,{ }^{* *} 1\end{array}$ & $\begin{array}{c}-15.3 \pm 1.9 \\
* 1\end{array}$ \\
\hline (p18) AHP (ms) & $\begin{array}{c}16.9 \pm 1.9 \\
-\end{array}$ & $\begin{array}{c}25.5 \pm 8.6 \\
-\end{array}$ & $\begin{array}{c}11.0 \pm 2.0 \\
-\end{array}$ & $\begin{array}{c}7.2 \pm 1.4 \\
-\end{array}$ & $\begin{array}{c}16.1 \pm 4.5 \\
-\end{array}$ \\
\hline (p19) ADP (mV) & $\begin{array}{c}0.3 \pm 0.1 \\
-\end{array}$ & $\begin{array}{c}1.3 \pm 0.8 \\
-\end{array}$ & $\begin{array}{c}0.0 \pm 0.0 \\
-\end{array}$ & $\begin{array}{c}0.0 \pm 0.0 \\
-\end{array}$ & $\begin{array}{c}0.0 \pm 0.0 \\
-\end{array}$ \\
\hline (p20) ADP (ms) & $\begin{array}{c}1.4 \pm 0.6 \\
-\end{array}$ & $\begin{array}{c}2.1 \pm 0.8 \\
-\end{array}$ & $\begin{array}{c}0.0 \pm 0.0 \\
-\end{array}$ & $\begin{array}{c}0.0 \pm 0.0 \\
-\end{array}$ & $\begin{array}{c}0.0 \pm 0.0 \\
-\end{array}$ \\
\hline (p21) Var A (\%o) & $\begin{array}{c}0.1 \pm 0.0 \\
-\end{array}$ & $\begin{array}{c}0.1 \pm 0.1 \\
-\end{array}$ & $\begin{array}{c}0.0 \pm 0.1 \\
-\end{array}$ & $\begin{array}{c}0.0 \pm 0.0 \\
-\end{array}$ & $\begin{array}{c}0.0 \pm 0.1 \\
-\end{array}$ \\
\hline (p22) Var D (\%o) & $\begin{array}{c}0.3 \pm 0.0 \\
-\end{array}$ & $\begin{array}{c}0.5 \pm 0.2 \\
-\end{array}$ & $\begin{array}{c}0.2 \pm 0.1 \\
-\end{array}$ & $\begin{array}{c}0.1 \pm 0.1 \\
-\end{array}$ & $\begin{array}{c}0.4 \pm 0.2 \\
-\end{array}$ \\
\hline$(p 23) I_{\max }(p A)$ & $\begin{array}{c}477.6 \pm 52.8 \\
-\end{array}$ & $\begin{array}{c}618.2 \pm 67.5 \\
-\end{array}$ & $\begin{array}{c}416.7 \pm 62.3 \\
-\end{array}$ & $\begin{array}{c}912.5 \pm 221.1 \\
-\end{array}$ & $\begin{array}{c}450.0 \pm 61.9 \\
-\end{array}$ \\
\hline (p24) Asat (Hz) & $\begin{array}{c}67.0 \pm 6.3 \\
* \star 2\end{array}$ & $\begin{array}{c}129.9 \pm 8.5 \\
{ }^{* *} 1,{ }^{* * *} 3,4\end{array}$ & $\begin{array}{c}47.7 \pm 5.7 \\
* \star \star 2\end{array}$ & $\begin{array}{c}31.9 \pm 9.3 \\
* \star * 2\end{array}$ & $\begin{array}{c}73.6 \pm 13.3 \\
-\end{array}$ \\
\hline (p25) Tsat (ms) & $\begin{array}{c}23.9 \pm 2.3 \\
-\end{array}$ & $\begin{array}{c}16.6 \pm 1.1 \\
{ }^{* *} 4\end{array}$ & $\begin{array}{c}22.0 \pm 4.2 \\
-\end{array}$ & $\begin{array}{c}94.0 \pm 37.8 \\
\star \star 2\end{array}$ & $\begin{array}{c}28.6 \pm 4.8 \\
-\end{array}$ \\
\hline$(\mathrm{p} 26) \mathbf{F}_{\max }(\mathrm{Hz})$ & $\begin{array}{l}55.2 \pm 2.3 \\
{ }^{\star} 3,{ }^{* *} 5,{ }^{* \star *} 4\end{array}$ & $\begin{array}{c}72.4 \pm 5.0 \\
-\end{array}$ & $\begin{array}{c}80.0 \pm 7.5 \\
* 1\end{array}$ & $\begin{array}{c}154.9 \pm 26.2 \\
* \star * 1\end{array}$ & $\begin{array}{c}93.9 \pm 9.6 \\
* * 1\end{array}$ \\
\hline$(\mathrm{p} 27) \mathbf{m}_{\mathrm{sat}}(\mathrm{Hz} / \mathrm{s})$ & $\begin{array}{c}-34.9 \pm 2.4 \\
{ }^{\star} 5,{ }^{* \star *} 3\end{array}$ & $\begin{array}{c}-33.4 \pm 3.5 \\
* \star 3\end{array}$ & $\begin{array}{c}-1.2 \pm 4.0 \\
* \star 2,{ }^{* \star *} 1\end{array}$ & $\begin{array}{c}-20.3 \pm 9.1 \\
-\end{array}$ & $\begin{array}{c}-10.2 \pm 6.2 \\
-\end{array}$ \\
\hline (p28) Hump (mV) & $\begin{array}{c}1.7 \pm 0.8 \\
* \star 2\end{array}$ & $\begin{array}{c}6.0 \pm 1.9 \\
{ }^{\star} 5,{ }^{\star \star} 1,4,{ }^{* \star *} 3\end{array}$ & $\begin{array}{c}0.5 \pm 0.3 \\
* \star \star 2\end{array}$ & $\begin{array}{c}0.0 \pm 0.0 \\
* \star 2\end{array}$ & $\begin{array}{c}2.0 \pm 2.0 \\
* 2\end{array}$ \\
\hline
\end{tabular}

$n$, Number of cells; Kruskal-Wallis and Dunn's multiple comparisons test. (pNumber) refer to the electrophysiological parameters described in the material et methods section. Asterisks indicate statistical significance $\left({ }^{*} P<0.05,{ }^{* *} P<0.01,{ }^{* * *} P<0.001\right)$ of the cluster in the column relative to the other clusters represented by their corresponding number.

\section{Miniature postsynaptic currents in wildtype mice}

To determine whether miniature postsynaptic currents (mIPSCs, mEPSCs) and the relative balance of excitation versus inhibition was different among the clusters, we simultaneously recorded mIPSCs and mEPSCs for each neuron of the clusters (Fig. 2). 

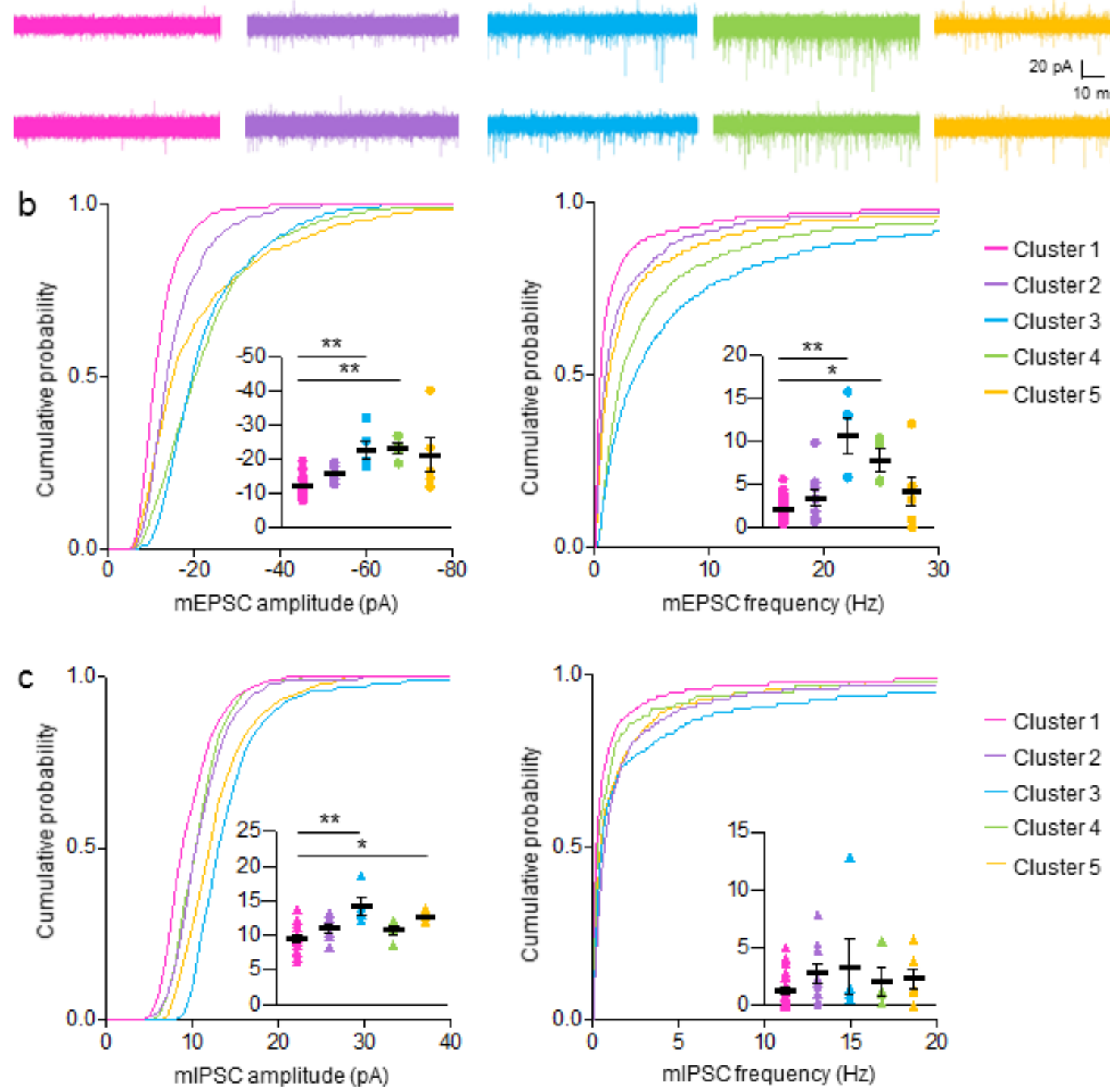

Figure. 2. Miniature IPSCs (mIPSCs) and EPSCs (mEPSCs) were recorded in each cluster in wildtype mice. (a) Representative recording from neurons of each cluster in the presence of $1 \mu \mathrm{M}$ TTX. (b) Cumulative probability and mean of both mEPSCs amplitude and frequency among the 5 clusters Kruskal-Wallis multiple comparisons test. (c) Cumulative probability and mean of mIPSCs amplitude and frequency among the 5 clusters Kruskal-Wallis multiple comparisons test.

mIPSCs were outward currents, whereas mEPSCs were inward currents at a holding potential of $-60 \mathrm{mV}$. Quantitative analysis revealed that mEPSCs recorded from neurons of cluster 1 were significantly smaller $(-12.1 \pm 0.5 \mathrm{pA}, n=25)$ and less frequent $(2.8 \pm 0.6 \mathrm{~Hz}, n=25)$ than neurons from cluster $3(-22.7 \pm 0.6 \mathrm{pA}, n=4$; $P=0.001$ Kruskal-Wallis multiple comparisons test $)(10.7 \pm 0.9 \mathrm{~Hz}, n=4 ; P=0.001$ 
Kruskal-Wallis multiple comparisons test) and $4(-23.2 \pm 1.6 \mathrm{pA}, n=4 ; \mathrm{P}=0.002$ Kruskal-Wallis multiple comparisons test) $(7.8 \pm 1.3 \mathrm{~Hz}, n=4$; $\mathrm{P}=0.036$ KruskalWallis test multiple comparisons test; Fig. 2b). In cluster 1, mean mIPSC amplitudes were smaller $(9.4 \pm 0.5 \mathrm{pA}, n=25)$ than those recorded in cluster $3(14.2 \pm 0.6 \mathrm{pA}$, $n=4 ; \mathrm{P}=0.003$ Kruskal-Wallis multiple comparisons test $)$ and $5(12.7 \pm 0.3 \mathrm{pA}, n=6$; $\mathrm{P}=0.031$ Kruskal-Wallis multiple comparisons test; Fig. 2c).

\section{Morphological properties of CA1 neurons in wildtype mice}

Diffusion of biocytin, a neuronal tracer contained in the patch pipette during electrophysiological recordings, permitted visualization of the recorded neurons (Fig. 3). Somatic and dendritic morphologies of the previously physiologically characterized neurons were successfully revealed for 32 out of the 62 recorded neurons. Their morphological variables were extracted from 3D Neurolucida reconstructions. Regarding basic morphometric analyses of somata, we found that there is no statistical difference between clusters (data not shown).

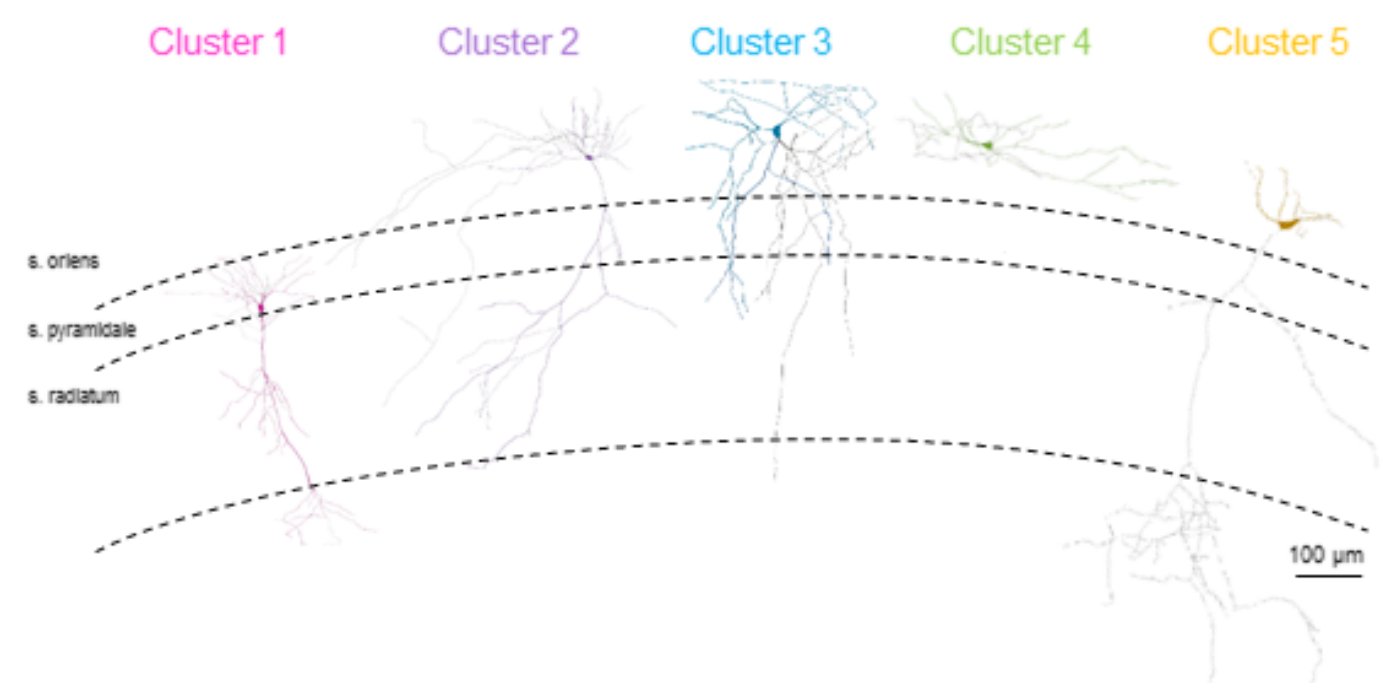

Figure. 3. Morphological features of CA1 neurons in wildtype mice. Neurolucida reconstructions of typical neurons from each cluster. Axons are represented in gray and dendrites are color-coded according to their cluster. 
We examined and compared the dendritic properties of CA1 neurons among the 5 neuronal clusters (Table 4). In particular, we found that neurons from cluster 5 are distinguished from neurons from cluster 1 and 2 by their simple and short dendritic arborization (p40, p41, p42, p44). Accordingly, their total length and ramifications at $300 \mu \mathrm{m}$ (p49) were significantly smaller compared to the other clusters (Table 4).

Table 4. Dendritic properties among CA1 neurons in wildtype mice

\begin{tabular}{|c|c|c|c|c|c|}
\hline & $\begin{array}{c}\text { Cluster } 1 \\
(n=16)\end{array}$ & $\begin{array}{c}\text { Cluster } 2 \\
(n=3)\end{array}$ & $\begin{array}{c}\text { Cluster } 3 \\
(n=5)\end{array}$ & $\begin{array}{c}\text { Cluster } 4 \\
(n=3)\end{array}$ & $\begin{array}{c}\text { Cluster } \mathbf{5} \\
(\mathrm{n}=5)\end{array}$ \\
\hline (p39) $\mathrm{Nb}$ of dendrites & $\begin{array}{c}4.8 \pm 0.4 \\
-\end{array}$ & $\begin{array}{c}5.7 \pm 0.8 \\
-\end{array}$ & $\begin{array}{c}5.8 \pm 1.4 \\
-\end{array}$ & $\begin{array}{c}4.3 \pm 1.3 \\
-\end{array}$ & $\begin{array}{c}3.8 \pm 0.2 \\
-\end{array}$ \\
\hline (p40) Total length $\left(10^{3} \mu \mathrm{m}\right)$ & $\begin{array}{l}4.4 \pm 0.5 \\
* \star 5\end{array}$ & $\begin{array}{l}5.1 \pm 1.2 \\
{ }^{*} 5\end{array}$ & $\begin{array}{c}3.1 \pm 0.8 \\
-\end{array}$ & $\begin{array}{c}1.8 \pm 0.3 \\
-\end{array}$ & $\begin{array}{l}0.9 \pm 0.3 \\
{ }^{*} 2,{ }^{* *} 1\end{array}$ \\
\hline (p41) Surface $\left(10^{3} \mu \mathrm{m}^{2}\right)$ & $\begin{array}{c}10.7 \pm 1.3 \\
* * 5\end{array}$ & $\begin{array}{c}13.7 \pm 2.9 \\
* 5\end{array}$ & $\begin{array}{c}6.9 \pm 1.9 \\
-\end{array}$ & $\begin{array}{c}4.5 \pm 0.8 \\
-\end{array}$ & $\begin{array}{l}2.1 \pm 0.7 \\
{ }^{*} 2,{ }^{* *} 1\end{array}$ \\
\hline (p42) Volume $\left(10^{3} \mu \mathrm{m}^{3}\right)$ & $\begin{array}{c}2.5 \pm 0.3 \\
1>5\end{array}$ & $\begin{array}{l}3.3 \pm 0.8 \\
* 5\end{array}$ & $\begin{array}{c}1.3 \pm 0.4 \\
-\end{array}$ & $\begin{array}{c}0.9 \pm 0.2 \\
-\end{array}$ & $\begin{array}{l}0.5 \pm 0.1 \\
* 1,2\end{array}$ \\
\hline (p43) Length/surface $\left(\mu \mathrm{m}^{-1}\right)$ & $\begin{array}{c}0.5 \pm 0.1 \\
-\end{array}$ & $\begin{array}{c}0.4 \pm 0.0 \\
-\end{array}$ & $\begin{array}{c}0.5 \pm 0.0 \\
-\end{array}$ & $\begin{array}{c}0.4 \pm 0.0 \\
-\end{array}$ & $\begin{array}{c}0.4 \pm 0.0 \\
-\end{array}$ \\
\hline $\begin{array}{l}\text { (p44) Highest order } \\
\text { segment }\end{array}$ & $\begin{array}{c}14.0 \pm 1.7 \\
* 5\end{array}$ & $\begin{array}{c}11.3 \pm 3.4 \\
-\end{array}$ & $\begin{array}{c}9 \pm 2.4 \\
-\end{array}$ & $\begin{array}{c}6.0 \pm 1.0 \\
-\end{array}$ & $\begin{array}{c}4.6 \pm 1.2 \\
* 1\end{array}$ \\
\hline (p45) Torsion ratio & $\begin{array}{c}1.1 \pm 0.0 \\
-\end{array}$ & $\begin{array}{c}1.0 \pm 0.0 \\
-\end{array}$ & $\begin{array}{c}1.0 \pm 0.0 \\
-\end{array}$ & $\begin{array}{c}1.1 \pm 0.0 \\
-\end{array}$ & $\begin{array}{c}1.0 \pm 0.0 \\
-\end{array}$ \\
\hline (p46) Nodes & $\begin{array}{c}33.3 \pm 3.4 \\
{ }^{*} 5\end{array}$ & $\begin{array}{c}30.3 \pm 7.4 \\
-\end{array}$ & $\begin{array}{c}27.4 \pm 9.4 \\
-\end{array}$ & $\begin{array}{c}12.3 \pm 2.8 \\
-\end{array}$ & $\begin{array}{c}7.2 \pm 1.9 \\
* 1\end{array}$ \\
\hline (p47) Sholl $100 \mu \mathrm{m}$ & $\begin{array}{c}0.3 \pm 0.0 \\
-\end{array}$ & $\begin{array}{c}0.3 \pm 0.0 \\
-\end{array}$ & $\begin{array}{c}0.4 \pm 0.1 \\
-\end{array}$ & $\begin{array}{c}0.4 \pm 0.1 \\
-\end{array}$ & $\begin{array}{c}0.6 \pm 0.1 \\
-\end{array}$ \\
\hline (p48) Sholl $200 \mu \mathrm{m}$ & $\begin{array}{c}0.3 \pm 0.0 \\
-\end{array}$ & $\begin{array}{c}0.1 \pm 0.0 \\
-\end{array}$ & $\begin{array}{c}0.3 \pm 0.1 \\
-\end{array}$ & $\begin{array}{c}0.3 \pm 0.1 \\
-\end{array}$ & $\begin{array}{c}0.1 \pm 0.0 \\
-\end{array}$ \\
\hline (p49) Sholl $300 \mu \mathrm{m}$ & $\begin{array}{c}0.1 \pm 0.0 \\
* 5\end{array}$ & $\begin{array}{c}0.1 \pm 0.0 \\
-\end{array}$ & $\begin{array}{c}0.2 \pm 0.0 \\
-\end{array}$ & $\begin{array}{c}0.1 \pm 0.1 \\
-\end{array}$ & $\begin{array}{c}0.0 \pm 0.0 \\
{ }^{*} 1\end{array}$ \\
\hline (p50) Sholl $400 \mu \mathrm{m}$ & $\begin{array}{c}0.2 \pm 0.0 \\
-\end{array}$ & $\begin{array}{c}0.4 \pm 0.2 \\
-\end{array}$ & $\begin{array}{c}0.1 \pm 0.1 \\
-\end{array}$ & $\begin{array}{c}0.2 \pm 0.1 \\
-\end{array}$ & $\begin{array}{c}0.1 \pm 0.1 \\
-\end{array}$ \\
\hline
\end{tabular}

$n$, Number of cells; Kruskal-Wallis and Dunn's multiple comparisons test. (pNumber) refer to the electrophysiological parameters described in the material et methods section. Asterisks indicate statistical significance $\left({ }^{*} P<0.05,{ }^{* *} P<0.01\right)$ of the cluster in the column relative to the other clusters represented by their corresponding number.

\section{Unsupervised classification of CA1 neurons from Cx30 deficient mice}

To determine whether Cx30 deficiency affects the electrophysiological and morphological properties of neurons from stratum oriens and stratum pyramidale, we 
repeated the previous characterization in WT on $\mathrm{C} \times 30^{\Delta / \Delta}$ mice. Forty-four neurons were characterized by patch-clamp recordings within the hippocampal CA1 stratum oriens and pyramidal of $\mathrm{C} \times 30^{\Delta / \Delta}$ mice. As performed on WT mice, the unsupervised Ward's clustering also revealed 5 neuronal clusters (Fig. 4).

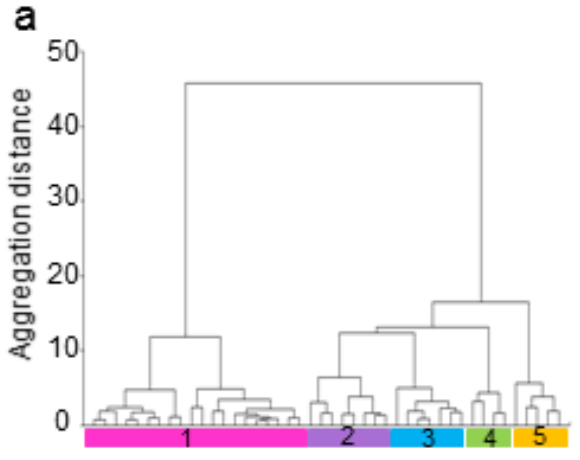

Cluster label b

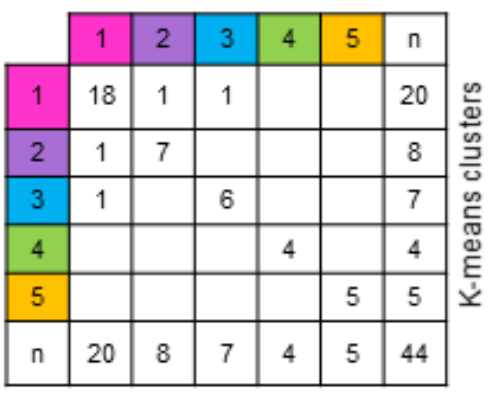

Ward clusters

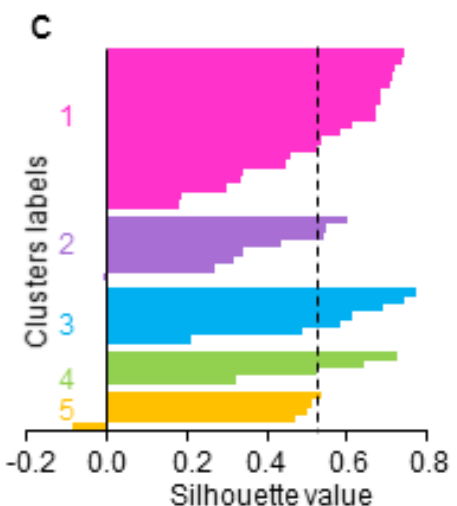

Cluster 4

Cluster 5

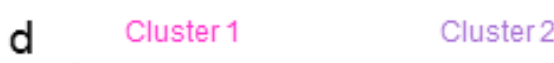

Cluster 3
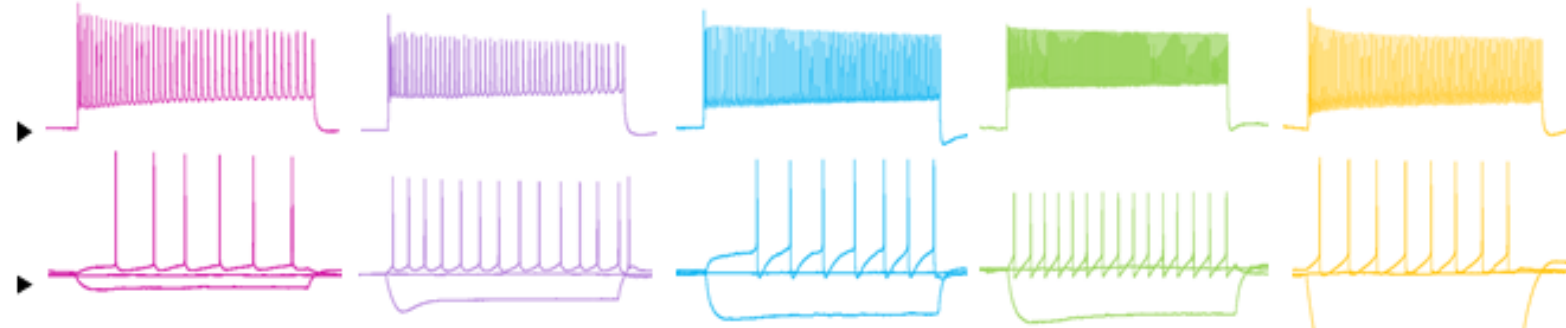

$50 \mathrm{mV}$
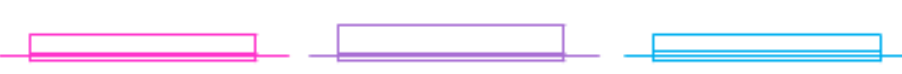

$300 \mathrm{pA} \mathrm{LL}_{10 \mathrm{~ms}}$

Figure 4. Unsupervised clustering of hippocampal CA1 neurons based on their electrophysiological properties recorded in $\mathrm{C} \times 30^{\Delta / \Delta}$ mice. (a) Ward's clustering of 44 CA1 neurons. Individual cells are represented along the $x$-axis. The $y$-axis represents the average within-cluster linkage distance in a space of 5 principal components extracted from 13 electrophysiological variables. Five clusters, 1 (in pink), 2 (in violet), 3 (in blue), 4 (in green) and 5 (in yellow) were identified. (b) Clusters generated by Ward's method in (a) were corrected using the clustering output generated by the K-means algorithm. (c) The silhouette analysis was used to assess the quality of the clustering that was optimal for 5 clusters with a mean value of 0.503 (dash line). (d) Typical electrophysiological behavior of neurons from each cluster recorded in current-clamp mode. Neurons from cluster 1 (pink), 2 (violet), 3 (blue), 4 (green) and 5 (yellow) were recorded using whole-cell patch-clamp recordings in response to the application of $800 \mathrm{~ms}$ current pulses (bottom traces). Strong depolarizing current evoked a high and sustained firing in all clusters (top traces). Typical voltage responses to hyperpolarizing current step injection (-100 pA), at resting 
membrane potential $(0 \mathrm{pA})$ and when depolarized at their spiking threshold evoked distinct responses in all clusters (middle traces).

The reliability of Ward's clustering was corrected using the K-mean algorithm. Two cells of cluster 1 were reassigned to cluster 2 and 3 , one cell of cluster 2 was reassigned to cluster 1 , and 1 cell from the cluster 3 was reassigned to cluster 1 (Fig. 4b). In order to determine whether the allocation of neurons into five groups was the optimal outcome of the clustering process, our results were compared with clustering trials whereby thresholds were set to define fewer or higher numbers of groups. We established that a cluster analysis yielding five groups was the best outcome with a mean silhouette value of 0.503 (Fig. 4b). Thus, the subdivision of CA1 neurons into five main groups remained in $\mathrm{C} \times 30^{\Delta / \Delta}$ mice.

The concordance of the clusters between the two classifications was confirmed by the maintenance of the typical characteristics of the cells in their belonging clusters. Indeed, the neurons from cluster 1 displayed the highest membrane capacitance ( $p 4$, Tables 1 and 5) in both classifications. The cells of cluster 2 always had an accommodative hump (p28, Tables 3 and 7 ). The cells from cluster 3 systematically had the most delayed first spike latency ( 10 , Tables 2 and 6). The interneurons of cluster 4 , had in both configurations the highest discharge frequency (p26, Tables 3 and 7). Similarly, for the neurons of cluster 5 , the Rhyp (p5, Tables 1 and 5) was always the most pronounced compared to the other neuronal clusters. 
Table 5. Comparison of passive membrane properties among CA1 neuron subtypes in $\mathrm{C} \times 30^{\Delta / \Delta}$ mice.

\begin{tabular}{|c|c|c|c|c|c|}
\hline & $\begin{array}{c}\text { Cluster } 1 \\
(\mathrm{n}=20)\end{array}$ & $\begin{array}{c}\text { Cluster } 2 \\
(n=8)\end{array}$ & $\begin{array}{c}\text { Cluster } 3 \\
(n=7)\end{array}$ & $\begin{array}{c}\text { Cluster } 4 \\
(n=4)\end{array}$ & $\begin{array}{c}\text { Cluster } 5 \\
(n=5)\end{array}$ \\
\hline (p1) RMP (mV) & $\begin{array}{c}-55.5 \pm 1.6 \\
-\end{array}$ & $\begin{array}{c}-54.6 \pm 1.6 \\
-\end{array}$ & $\begin{array}{c}-52.9 \pm 2.5 \\
-\end{array}$ & $\begin{array}{c}-51.5 \pm 1.1 \\
-\end{array}$ & $\begin{array}{c}-52.2 \pm 1.2^{\star} \\
-\end{array}$ \\
\hline (p2) $\mathbf{R}_{\mathbf{m}}(\mathrm{M} \Omega)$ & $\begin{array}{c}355.4 \pm 31.9 \\
* \star \star 5\end{array}$ & $\begin{array}{c}539.2 \pm 63.7^{\star \star} \\
-\end{array}$ & $\begin{array}{c}498.2 \pm 78.9 \\
-\end{array}$ & $\begin{array}{c}368.7 \pm 136.8 \\
{ }^{*} 5\end{array}$ & $\begin{array}{c}1267.8 \pm 161.3 \\
{ }^{*} 4,{ }^{* * *} 1\end{array}$ \\
\hline (p4) $T_{m}(m s)$ & $\begin{array}{c}27.7 \pm 2.4 \\
-\end{array}$ & $\begin{array}{c}30.4 \pm 5.2^{*} \\
-\end{array}$ & $\begin{array}{c}21.5 \pm 1.6 \\
* 5\end{array}$ & $\begin{array}{c}17.5 \pm 5.8 \\
* 5\end{array}$ & $\begin{array}{c}65.4 \pm 14.5 \\
* 3,4\end{array}$ \\
\hline$(p 4) C_{m}(p F)$ & $\begin{array}{c}81.6 \pm 4.8 \\
* 3\end{array}$ & $\begin{array}{c}58.6 \pm 7.1 \\
-\end{array}$ & $\begin{array}{l}48.8 \pm 6.9 \\
* 1\end{array}$ & $\begin{array}{c}49.9 \pm 5.4 \\
-\end{array}$ & $\begin{array}{c}50.9 \pm 8.7 \\
-\end{array}$ \\
\hline (p5) $\mathbf{R}_{\text {hyp }}(\mathrm{M} \Omega$ ) & $\begin{array}{c}264.9 \pm 21.5 \\
\star 2,{ }^{* \star *} 5\end{array}$ & $\begin{array}{c}\mathbf{4 6 0 . 2} \pm \mathbf{3 0 . 9 ^ { \star \star \star }} \\
{ }^{* 1}\end{array}$ & $\begin{array}{c}454.2 \pm 78.3^{*} \\
-\end{array}$ & $\begin{array}{c}337.3 \pm 101.9 \\
-\end{array}$ & $\underset{* \star \star 1}{772.9 \pm 89.3}$ \\
\hline (p6) $\mathbf{R}_{\text {sag }}(\mathrm{M} \Omega)$ & $\underset{\star * \star 5}{212.9 \pm 17.2}$ & $\begin{array}{c}279.1 \pm 20.2^{\star *} \\
-\end{array}$ & $\begin{array}{c}391.6 \pm 69.7 \\
-\end{array}$ & $\begin{array}{c}288.8 \pm 85.4 \\
-\end{array}$ & $\underset{* * * 1}{665.3 \pm 86.3}$ \\
\hline (p7) $\Delta \mathbf{R}_{\text {sag }}(\%)$ & $\begin{array}{c}80.5 \pm 1.1 \\
* 2\end{array}$ & $\begin{array}{c}60.6 \pm 1.6^{* \star *} \\
{ }^{*} 1,{ }^{* *} 4,5,{ }^{* \star \star} 3\end{array}$ & $\begin{array}{c}85.9 \pm 2.0^{*} \\
* \star \star 2\end{array}$ & $\begin{array}{c}86.4 \pm 1.6 \\
* * 2\end{array}$ & $\begin{array}{c}85.9 \pm 3.1 \\
* \star 2\end{array}$ \\
\hline
\end{tabular}

$n$, Number of cells; Kruskal-Wallis and Dunn's multiple comparisons test. Statistically different values from wildtype cluster are written in bold, Mann-Whitney U-test. (pNumber) refer to the electrophysiological parameters described in the material et methods section. Asterisks indicate statistical significance $\left({ }^{*} P<0.05,{ }^{* *} P<0.01\right.$, ${ }^{* * *} P<0.01$ ) of the cluster in the column relative to the other clusters represented by their corresponding number.

Table 6. Comparison of just above threshold properties among CA1 neuron subtypes in $\mathrm{C} \times 30^{\Delta / \Delta}$ mice.

\begin{tabular}{|c|c|c|c|c|c|}
\hline & $\begin{array}{c}\text { Cluster } 1 \\
(\mathrm{n}=20)\end{array}$ & $\begin{array}{c}\text { Cluster } 2 \\
(\mathrm{n}=8)\end{array}$ & $\begin{array}{c}\text { Cluster } 3 \\
(n=7)\end{array}$ & $\begin{array}{c}\text { Cluster } 4 \\
(n=4)\end{array}$ & $\begin{array}{c}\text { Cluster } 5 \\
(n=5)\end{array}$ \\
\hline$(\mathrm{p} 8) \mathbf{A P}_{\mathrm{th}}(\mathrm{mV})$ & $\begin{array}{c}-48.9 \pm 0.9^{*} \\
* \star 3\end{array}$ & $\begin{array}{c}-45.5 \pm 2.3^{*} \\
-\end{array}$ & $\begin{array}{c}-38.0 \pm 1.1^{\star \star} \\
\star \star 1\end{array}$ & $\begin{array}{c}-42.3 \pm 4.2 \\
-\end{array}$ & $\begin{array}{c}-43.7 \pm 3.2 \\
-\end{array}$ \\
\hline (p9) Rheobase (pA) & $\begin{array}{c}22.0 \pm 3.4 \\
-\end{array}$ & $\begin{array}{c}18.8 \pm 5.2 \\
-\end{array}$ & $\begin{array}{c}38.6 \pm 7.0 \\
* 5\end{array}$ & $\begin{array}{c}85.0 \pm 59.5 \\
-\end{array}$ & $\begin{array}{c}12.0 \pm 2.0 \\
* 3\end{array}$ \\
\hline $\begin{array}{c}(\mathrm{p} 10) \mathbf{1}^{\text {st }} \text { spike } \\
(\mathrm{ms})\end{array}$ & $\begin{array}{c}147.7 \pm 27.5 \\
* 3\end{array}$ & $\begin{array}{c}82.7 \pm 11.6 \\
\star * 3\end{array}$ & $\begin{array}{c}\mathbf{4 8 7 . 6} \mathbf{\pm} \mathbf{6 8 . 9} . \mathbf{9}^{\star} \\
{ }^{*} 1,{ }^{* \star} 5,2\end{array}$ & $\begin{array}{c}211.5 \pm 111.1 \\
-\end{array}$ & $\underset{* \star 3}{126.2 \pm 87.8}$ \\
\hline$(\mathrm{p} 11) \mathbf{m}_{\mathrm{th}}(\mathrm{Hz} / \mathrm{s})$ & $\begin{array}{c}-3.0 \pm 1.5^{\star} \\
* * 3\end{array}$ & $\begin{array}{c}0.1 \pm 3.2 \\
* 3\end{array}$ & $\begin{array}{c}7.4 \pm 1.9^{\star * \star} \\
{ }^{*} 2,{ }^{* \star} 1,4\end{array}$ & $\begin{array}{c}-14.4 \pm 7.7 \\
* * 3\end{array}$ & $\begin{array}{c}-1.6 \pm 1.0 \\
-\end{array}$ \\
\hline$(\mathrm{p} 12) \mathbf{F}_{\mathrm{th}}(\mathrm{Hz})$ & $\begin{array}{c}9.5 \pm 1.4 \\
-\end{array}$ & $\begin{array}{c}7.1 \pm 1.1 \\
-\end{array}$ & $\begin{array}{c}8.2 \pm 0.7 \\
-\end{array}$ & $\begin{array}{c}23.6 \pm 13.4 \\
-\end{array}$ & $\begin{array}{c}12.2 \pm 2.6 \\
-\end{array}$ \\
\hline
\end{tabular}

$n$, Number of cells; Kruskal-Wallis and Dunn's multiple comparisons test. Statistically different values from WT cluster are written in bold, Mann-Whitney U-tests. (pNumber) refer to the electrophysiological parameters described in the material et methods section. 
Table 7. Comparison of action potential properties among CA1 neurons in $\mathrm{C} \times 30^{\Delta / \Delta}$ mice.

\begin{tabular}{|c|c|c|c|c|c|}
\hline & $\begin{array}{l}\text { Cluster } 1 \\
(n=20)\end{array}$ & $\begin{array}{c}\text { Cluster } 2 \\
(n=8)\end{array}$ & $\begin{array}{c}\text { Cluster } 3 \\
(n=7)\end{array}$ & $\begin{array}{c}\text { Cluster } 4 \\
(n=4)\end{array}$ & $\begin{array}{c}\text { Cluster } 5 \\
(n=5)\end{array}$ \\
\hline (p13) A1 (mV) & $\begin{array}{c}100.5 \pm 1.6^{\star} \\
{ }^{\star *} 3,5,{ }^{* \star} 4\end{array}$ & $\begin{array}{c}83.7 \pm 3.9 \\
-\end{array}$ & $\begin{array}{c}74.7 \pm 3.0 \\
* * 1\end{array}$ & $\begin{array}{c}59.5 \pm 4.7 \\
* \star \star 1\end{array}$ & $\begin{array}{c}73.2 \pm 8.3 \\
* * 1\end{array}$ \\
\hline (p14) D1 (ms) & $\begin{array}{c}1.3 \pm 0.0^{\star *} \\
{ }^{\star *} 2,5,{ }^{* \star *} 4\end{array}$ & $\begin{array}{c}0.9 \pm 0.0^{*} \\
* 1\end{array}$ & $\begin{array}{c}0.9 \pm 0.1 \\
-\end{array}$ & $\begin{array}{c}0.6 \pm 0.0 \\
* * * 1\end{array}$ & $\begin{array}{c}0.9 \pm 0.1 \\
* \star 2\end{array}$ \\
\hline (p15) A2 (mV) & $\begin{array}{l}99.1 \pm 1.5^{\star} \\
{ }^{\star} 2,5,{ }^{\star \star} 3,{ }^{* \star *} 4\end{array}$ & $\begin{array}{c}82.3 \pm 3.9 \\
* 1\end{array}$ & $\begin{array}{c}74.9 \pm 2.8 \\
* * 1\end{array}$ & $\begin{array}{c}63.8 \pm 3.8 \\
* \star \star 1\end{array}$ & $\begin{array}{c}76.3 \pm 7.8 \\
* 1\end{array}$ \\
\hline (p16) D2 (ms) & $\begin{array}{c}1.3 \pm 0.0^{\star \star} \\
{ }^{\star *} 2,5,{ }^{* \star *} 4\end{array}$ & $\begin{array}{c}0.9 \pm 0.0^{\star \star \star} \\
\star \star\end{array}$ & $\begin{array}{c}1.0 \pm 0.1 \\
-\end{array}$ & $\begin{array}{c}0.6 \pm 0.1 \\
* \star * \\
0\end{array}$ & $\begin{array}{c}0.9 \pm 0.1 \\
* \star 1\end{array}$ \\
\hline (p17) AHP (mV) & $\begin{array}{l}-5.5 \pm 0.8 \\
{ }^{\star *} 4,5,{ }^{* \star *} 3\end{array}$ & $\begin{array}{c}-15.1 \pm 1.8^{\star \star \star} \\
-\end{array}$ & $-\underbrace{\star \star \star} 1$ & $\underset{* \star 1}{-23.6 \pm 1.9}$ & $\begin{array}{c}-21.4 \pm 2.9 \\
* \star 1\end{array}$ \\
\hline (p18) AHP (ms) & $\begin{array}{c}9.7 \pm 3.5^{\star \star} \\
-\end{array}$ & $\begin{array}{c}13.0 \pm 3.9 \\
-\end{array}$ & $\begin{array}{c}12.4 \pm 0.4 \\
-\end{array}$ & $\begin{array}{c}5.9 \pm 1.3 \\
-\end{array}$ & $\begin{array}{c}8.3 \pm 1.1 \\
-\end{array}$ \\
\hline (p19) ADP (mV) & $\begin{array}{c}0.4 \pm 0.2 \\
-\end{array}$ & $\begin{array}{c}0.8 \pm 0.8 \\
-\end{array}$ & $\begin{array}{c}0.0 \pm 0.0 \\
-\end{array}$ & $\begin{array}{c}0.0 \pm 0.0 \\
-\end{array}$ & $\begin{array}{c}0.0 \pm 0.0 \\
-\end{array}$ \\
\hline (p20) ADP (ms) & $\begin{array}{c}1.1 \pm 0.4 \\
-\end{array}$ & $\begin{array}{c}0.7 \pm 0.7 \\
-\end{array}$ & $\begin{array}{c}0.0 \pm 0.0 \\
-\end{array}$ & $\begin{array}{c}0.0 \pm 0.0 \\
-\end{array}$ & $\begin{array}{c}0.0 \pm 0.0 \\
-\end{array}$ \\
\hline$(p 21) \operatorname{Var} \mathbf{A}(\%)$ & $\begin{array}{c}0.8 \pm 0.4^{\star \star \star} \\
-\end{array}$ & $\begin{array}{c}0.1 \pm 0.0 \\
-\end{array}$ & $\begin{array}{c}0.1 \pm 0.1 \\
-\end{array}$ & $\begin{array}{c}0.7 \pm 0.4^{\star} \\
-\end{array}$ & $\begin{array}{c}0.1 \pm 0.1^{*} \\
-\end{array}$ \\
\hline (p22) Var D (\%o) & $\begin{array}{c}0.1 \pm 0.4^{\star \star} \\
-\end{array}$ & $\begin{array}{c}0.1 \pm 0.1 \\
-\end{array}$ & $\begin{array}{c}0.8 \pm 0.5 \\
-\end{array}$ & $\begin{array}{c}0.3 \pm 0.2 \\
-\end{array}$ & $\begin{array}{c}0.6 \pm 1.5 \\
-\end{array}$ \\
\hline$(p 23) I_{\max }(p A)$ & $\begin{array}{c}385.0 \pm 50.7 \\
* 4\end{array}$ & $\begin{array}{c}500.0 \pm 35.3 \\
-\end{array}$ & $\begin{array}{c}571.4 \pm 56.5 \\
-\end{array}$ & $\begin{array}{c}1000.0 \pm 181.4 \\
*_{1}\end{array}$ & $\begin{array}{c}380.0 \pm 37.4 \\
-\end{array}$ \\
\hline (p24) $A_{\text {sat }}(\mathrm{Hz})$ & $\begin{array}{c}71.2 \pm 6.9 \\
-\end{array}$ & $\begin{array}{c}75.0 \pm 12.7^{\star \star} \\
-\end{array}$ & $\begin{array}{c}86.9 \pm 14.1^{*} \\
-\end{array}$ & $\begin{array}{c}58.1 \pm 4.4^{\star} \\
-\end{array}$ & $\begin{array}{c}47.4 \pm 4.4 \\
-\end{array}$ \\
\hline (p25) Tsat (ms) & $\begin{array}{c}23.4 \pm 3.5 \\
-\end{array}$ & $\begin{array}{c}30.1 \pm 9.4 \\
-\end{array}$ & $\begin{array}{c}10.9 \pm 0.9 \\
\quad 3<4\end{array}$ & $\begin{array}{c}33.4 \pm 5.6 \\
* 3\end{array}$ & $\begin{array}{c}24.6 \pm 5.4 \\
-\end{array}$ \\
\hline$(\mathrm{p} 26) \mathbf{F}_{\max }(\mathrm{Hz})$ & $\begin{array}{c}\mathbf{6 1 . 7} \pm \mathbf{2 . 6} \mathbf{6}^{\star} \\
{ }^{\star *} 2,5,{ }^{\star \star *} 4\end{array}$ & $\underset{ }{100.7 \pm 7.9^{\star * *}}$ & $\begin{array}{c}73.6 \pm 7.3 \\
-\end{array}$ & $\underset{* * * 1}{167.8 \pm 19.5}$ & $\underset{* * 1}{116.3 \pm 16.8}$ \\
\hline$(\mathrm{p} 27) \mathbf{m}_{\text {sat }}(\mathrm{Hz} / \mathrm{s})$ & $\begin{array}{c}-38.1 \pm 2.9 \\
{ }^{*} 4,{ }^{* \star *} 3\end{array}$ & $\begin{array}{c}-11.5 \pm 7.5^{\star} \\
-\end{array}$ & $\begin{array}{c}16.9 \pm 4.5^{\star \star} \\
\star \star \star 1\end{array}$ & $\begin{array}{c}-1.7 \pm 4.7 \\
* 1\end{array}$ & $\begin{array}{c}-8.7 \pm 2.3 \\
-\end{array}$ \\
\hline (p28) Hump (mV) & $\begin{array}{c}1.3 \pm 0.4 \\
-\end{array}$ & $\begin{array}{c}1.6 \pm 1.3^{*} \\
- \\
\end{array}$ & $\begin{array}{c}1.3 \pm 0.4^{\star} \\
- \\
\end{array}$ & $\begin{array}{c}0.0 \pm 0.0 \\
-\end{array}$ & $\begin{array}{c}0.3 \pm 0.3 \\
-\end{array}$ \\
\hline
\end{tabular}

$n$, Number of cells; Kruskal-Wallis, followed by a Dunn's multiple comparisons test. Statistically different values from cluster in WT mice are written in bold, Mann-Whitney $U$-test. Asterisks indicate statistical significance $\left({ }^{*} P<0.05,{ }^{* *} P<0.01,{ }^{* * *} P<0.01\right)$ of the cluster in the column relative to the other clusters represented by their corresponding number.

Electrophysiological Diversity of the hippocampal CA1 Stratum Oriens and Pyramidal Neurons in Cx30 deficient mice.

Although the 5 identified clusters in $C \times 30^{\Delta / \Delta}$ mice generally retained the same properties than in WT mice, we also observed electrophysiological differences among 
these clusters. Regarding the passive membrane properties, the RMP ( $p 1$ ) of neurons from cluster 5 of $C \times 30^{\Delta / \Delta}$ mice were more depolarized (from $-58.7 \pm 1.9$ to $52.2 \pm 1.2 \mathrm{mV}, \mathrm{P}=0.03$, Mann-Whitney U-test; Table 1 and 6; Fig. 5a,b). A significant lower membrane resistance was observed for cluster 2 neurons, whose passive membrane properties are the most affected by the absence of $\mathrm{C} \times 30$. Indeed, their membrane resistance (p2) was also significantly higher, as their Ih current, leading to a higher membrane time constant (p4), steeper slope (p5, p6) and a smaller sag ratio (p7) compared to other groups (Table 5). Neurons from cluster 3 also displayed a significant smaller sag ratio in $\mathrm{C} \times 30^{\Delta / \Delta}$ mice (p7, Fig. $5 \mathrm{c}, \mathrm{d}$ ).

Regarding the properties of neurons when depolarized just above threshold, we observed that compared to WT, in $C \times 30^{\Delta / \Delta}$ mice, neurons have globally a higher action potential threshold (p8; from $-64.1 \pm 0.8$ to $-55.2 \pm 0.9 \mathrm{mV}, \mathrm{P}=0.0005$, Mann-Whitney U-test, Fig. 5e,f). This was mostly observed for neurons from cluster 1, 2 and 3 (Table 6; Fig. 5e,f). In particular, Cx30 deficiency strongly affected neurons from cluster 3 , which have a significantly increased action potential threshold ( $p 8, \sim 15 \%$ ), $1^{\text {st }}$ spike latency $(\mathrm{p} 10, \sim 93 \%)$ and firing frequency adaptation at threshold ( $\mathrm{p} 11$, $+208 \%$; Tables 2 and 6), suggesting that these neurons are less excitable when depolarized near threshold. 

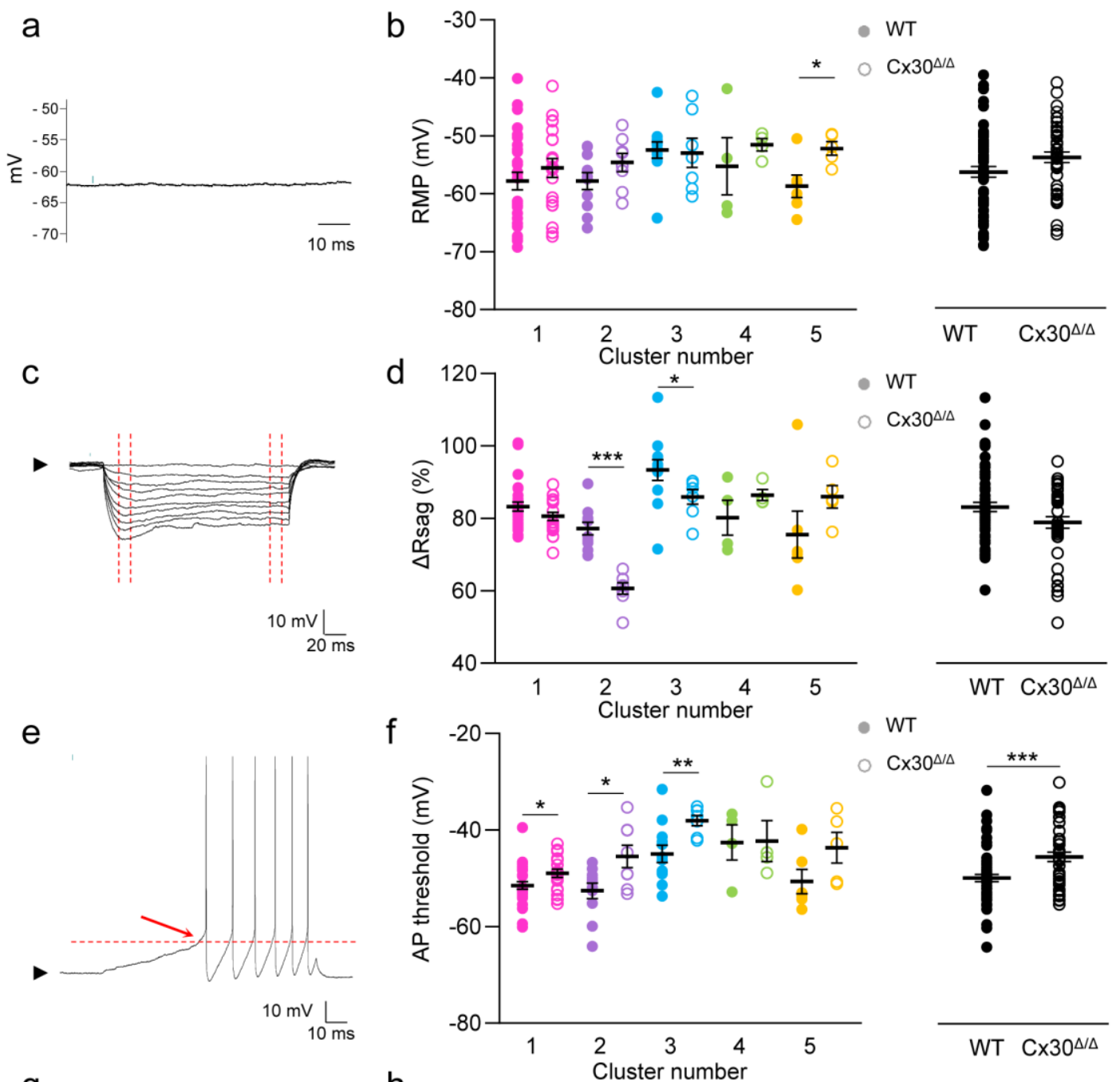

- WT
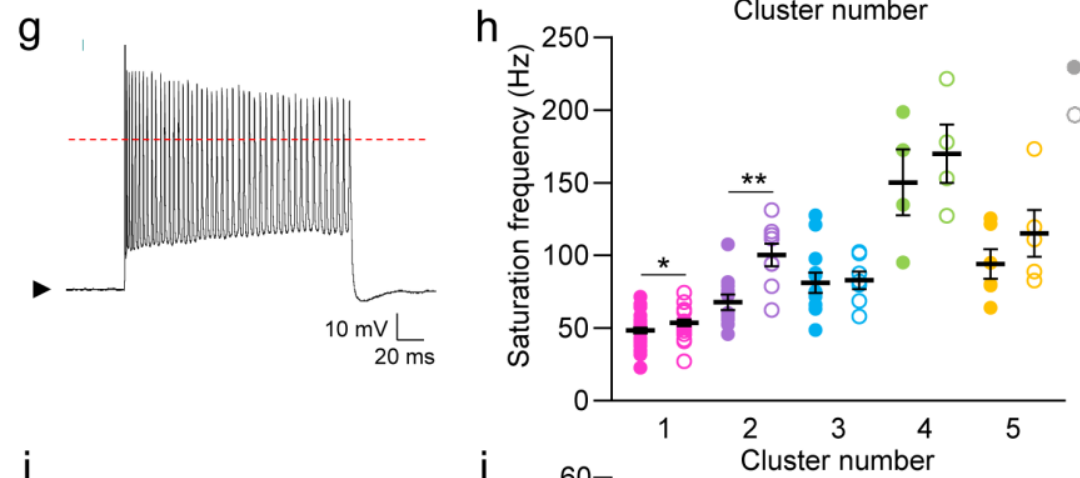

$C \times 30^{\Delta / \Delta}$
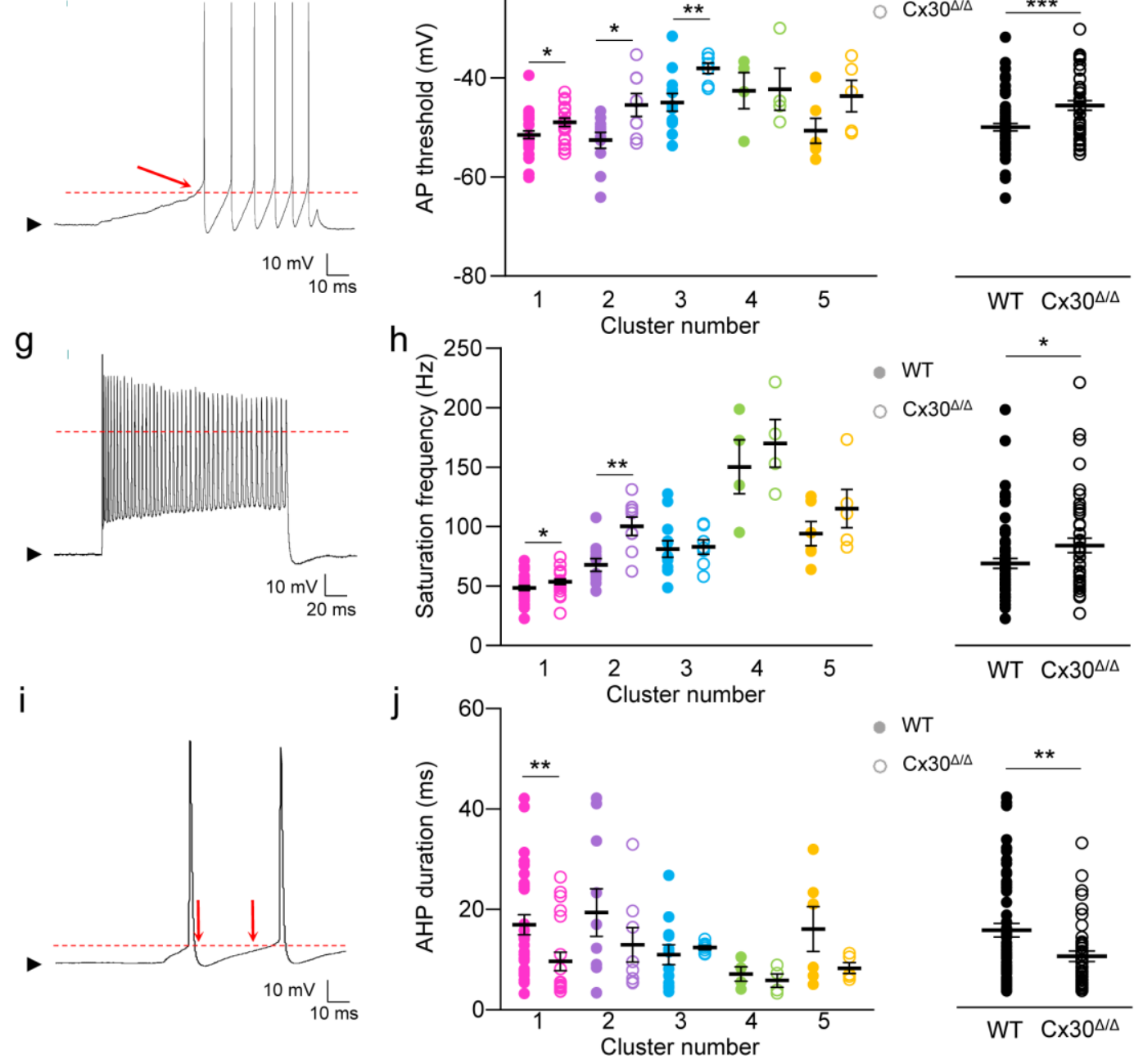

Figure 5. Main electrophysiological properties significantly different in Cx30 $\Delta / \Delta$ compared to wildtype mice. (a) Representative trace showing the membrane 
potential measurement. (b) Dot plot representing the membrane potential among the 5 clusters in WT and $\mathrm{C} \times 30^{\Delta / \Delta}$ mice (Mann-Whitney U-test, left panel), and globally in WT and $\mathrm{C} \times 30^{\Delta / \Delta}$ mice (t-test, right panel). (c) Representative trace showing the $\Delta$ Rsag measurement. (d) Dot plot representing the $\Delta$ Rsag among the 5 clusters in WT and

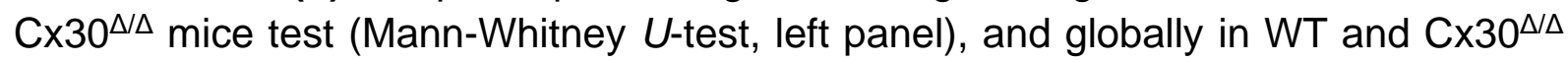
mice (t-test, right panel). (e) Representative trace showing the action potential threshold measurement. (f) Dot plot representing the action potential threshold among the 5 clusters in WT and $\mathrm{C} \times 30^{\Delta / \Delta}$ mice (Mann-Whitney U-test, left panel), and globally in WT and $\mathrm{C} \times 30^{\Delta / \Delta}$ mice (t-test, right panel). (g) Representative trace showing the saturation frequency measurement. (h) Dot plot representing the saturation frequency among the 5 clusters in WT and $\mathrm{C} \times 30^{\Delta / \Delta}$ mice (Mann-Whitney U-test, left panel), and globally in WT and $\mathrm{C} \times 30^{\Delta / \Delta}$ mice (t-test, right panel). I, Representative trace showing the AHP duration measurement. (h) Dot plot representing the AHP duration among the 5 clusters in WT and $\mathrm{C} \times 30^{\Delta / \Delta}$ mice (Mann-Whitney U-test, left panel), and globally in WT and $C \times 30^{\Delta / \Delta}$ mice (t-test, right panel).

Concerning action potential properties in $C \times 30^{\Delta / \Delta}$ mice, we found that neurons from clusters 1 and 2 were particularly affected. In cluster 1 neurons, action potential amplitudes (for both $p 13$ and p15, Tables 3 and 7) were significantly increased, by $9 \%$, whereas their duration were reduced by $13 \%$ (for both $\mathrm{p} 14$ and $\mathrm{p} 16$ ). Neurons from cluster 2 also have decreased action potential duration by 18\% (for both $\mathrm{p} 14$ and $\mathrm{p} 16$, Tables 3 and 7). We observed that in $C \times 30^{\Delta / \Delta}$ mice, neurons have globally a higher saturation frequency (p26) by $\sim 22 \%$ This was observed in particular in neurons from cluster 1 and 2, which increased their saturation frequency by $\sim 12 \%$ and $\sim 39 \%$ respectively (Tables 3 and 7; Fig. 5g,h). We also observed that in $C \times 30^{\Delta / \Delta}$ mice, neurons overall have a shorter AHP duration by $\sim 49 \%$. This was observed in particular in neurons from cluster 1 with a reduction of $\sim 57 \%$ (Tables 7; Fig. 5i,j). Moreover, AHP amplitudes were also increased in neurons, from cluster 2 to 5 , by $42 \%$. These results indicate that despite a reduced neuronal excitability at threshold, they can sustain higher firing frequency by modulating their AHP properties. 


\section{Miniature Postsynaptic Currents in Cx30 deficient mice}

To determine whether the balance between excitatory and inhibitory synaptic transmission was altered $\mathrm{C} \times 30^{\Delta / \Delta}$ mice, mEPSCs and mIPSCs were measured from individual neurons of each cluster at a holding potential of $-60 \mathrm{mV}$ (Fig. 6a). Quantitative analysis showed that the cumulative probability of mEPSCs amplitude is significantly increased in all clusters, except for cluster 3 , where on the opposite, a decrease is measured (Fig. 6b). Instantaneous frequency of mEPSCs further revealed a significant decreased frequency in cluster 1 and 3 , a significant increase in cluster 2 and 4, and no effect on cluster 5 neurons. No differences were found regarding cumulative probability of mIPSCs amplitudes between $C \times 30^{\Delta / \Delta}$ and WT mice. Yet, cumulative probability of mIPSCs frequencies were significantly increased in cluster 1 and 4 and significantly decreased in the three other clusters (Fig. 6c).

To determine whether the balance between excitatory and inhibitory synaptic transmission was altered in $C \times 30^{\Delta / \Delta}$ mice, we compared the mean ratio of frequency and amplitude of mEPSCs to mIPSCs in all clusters from Cx30 deficient and WT mice (Fig. 6c). We found that the mean ratio of frequency is significantly decreased in cluster 1 neurons (from $2.1 \pm 0.1$ to $0.7 \pm 0.1 \mathrm{mV}, P<0.0001$, t-test), indicating that the balance between excitation and inhibition was shifted to favor inhibition over excitation. On the opposite, this ratio of frequency is significantly increased in cluster 2 neurons (from $1.2 \pm 0.2$ to $2.7 \pm 0.4 \mathrm{mV}, \mathrm{P}=0.009$, t-test), indicating a shift toward excitation. No effect was observed regarding the mean ratio of frequency in the other clusters. 

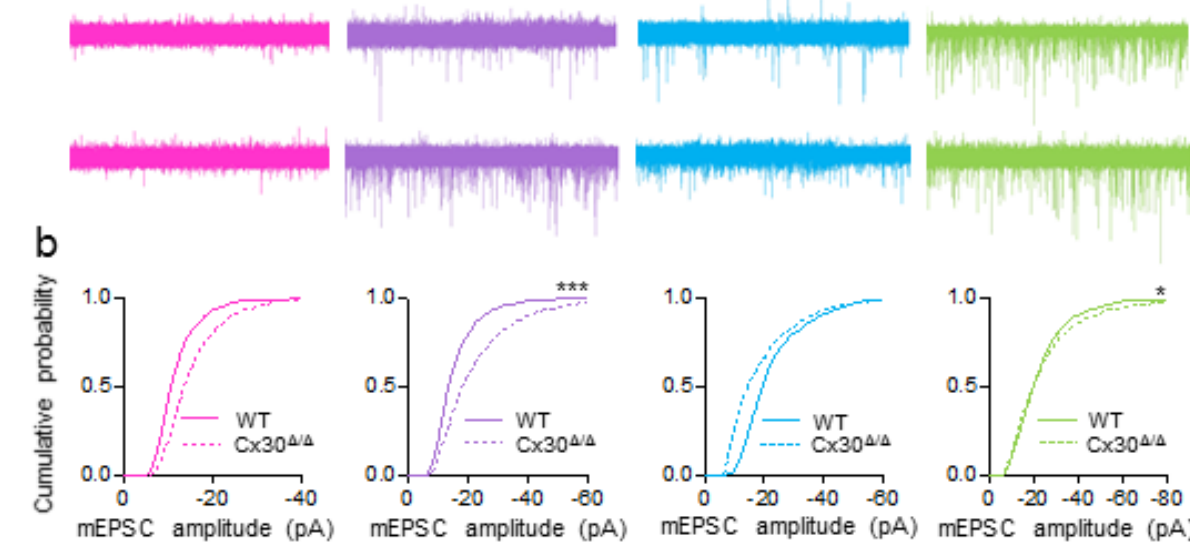

$20 \mathrm{pA} \frac{\mathrm{L}}{10 \mathrm{~ms}}$
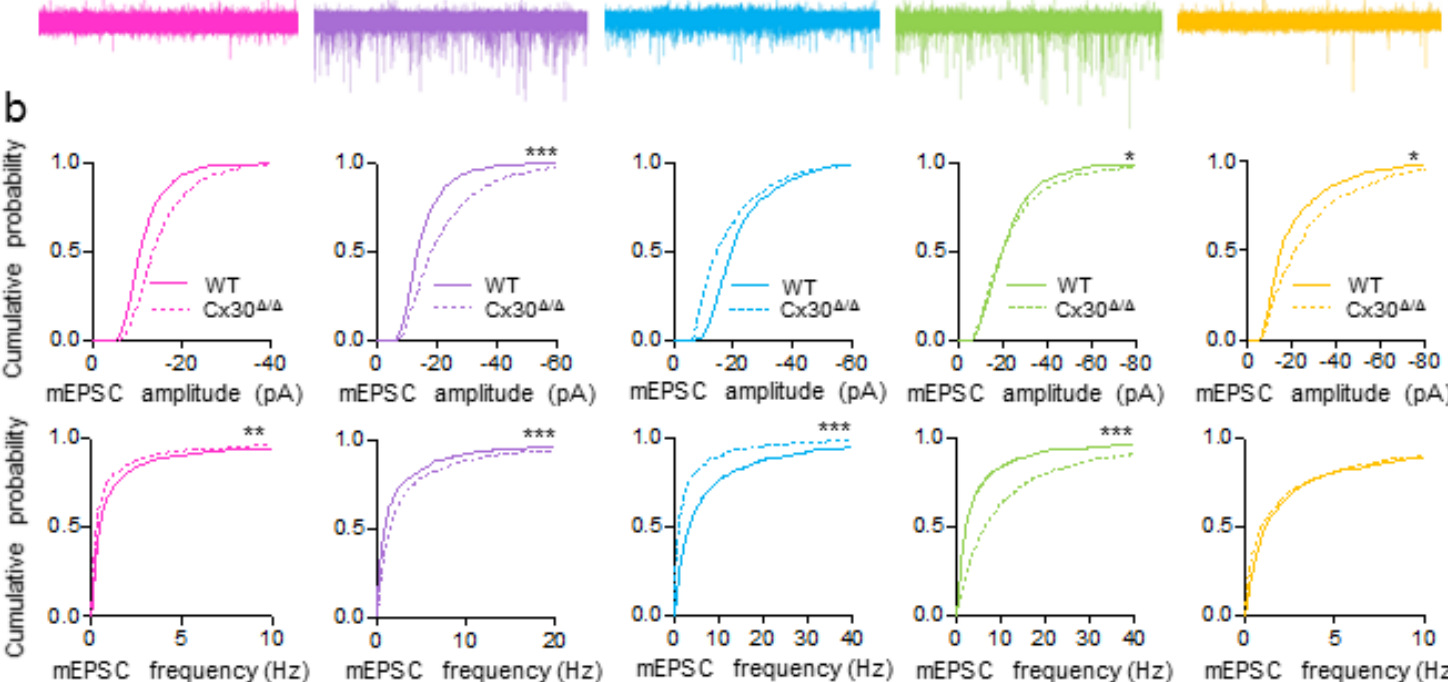

mEPSC amplitude ( $\mathrm{pA}$ )
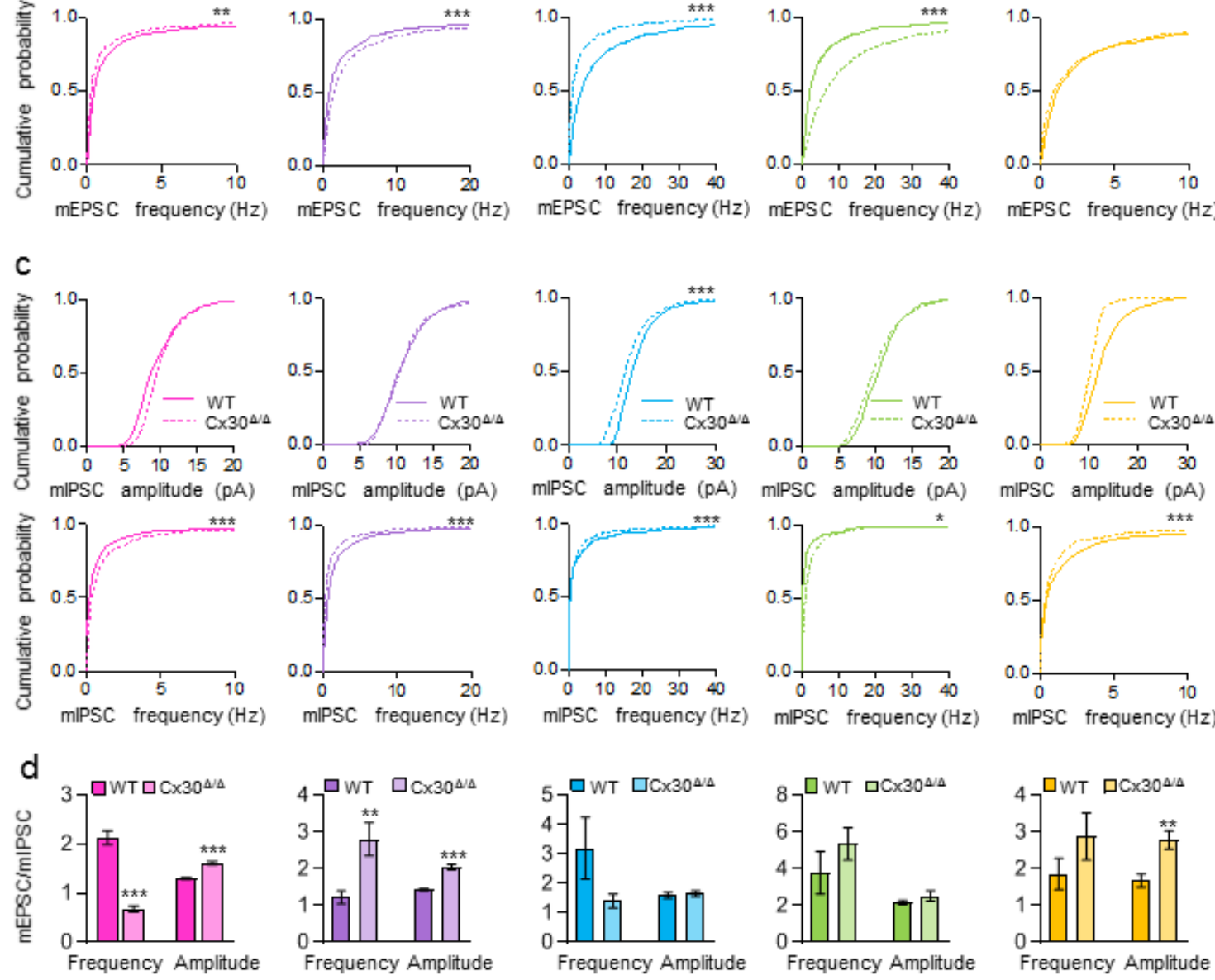

Figure 6. Miniature IPSCs (mIPSCs) and EPSCs (mEPSCs) in each cluster in Cx30 deficient mice. (a) Representative recordings from neurons of each cluster in the presence of $1 \mu \mathrm{M} \mathrm{TTX}$. (b) Cumulative probability of mEPSCs amplitude and frequency comparison between WT and $\mathrm{C} \times 30^{\Delta / \Delta}$ mice for each cluster, Kolmogorov-Smirnov test. (c) Cumulative probability of mIPSCs amplitude and frequency comparison between WT and $\mathrm{C} \times 30^{\Delta / \Delta}$ mice for each cluster Kolmogorov-Smirnov test. (d) mEPSCs/mIPSCs amplitude and frequency comparison between WT and $C \times 30^{\Delta / \Delta}$ mice for each cluster ratio, t-test. 
Regarding the ratio of mEPSCs to mIPSCs amplitudes, we found an increased ratio in cluster 1,2 and 5 by $\sim 23 \%, \sim 43 \%$ and $\sim 65 \%$ respectively. No effect was observed regarding the mean ratio of amplitude in the other clusters (Fig. 6d). Cx30 deficiency thus mainly increases the E/I balance on cluster 2 neurons, and has opposite effect on pyramidal cells of cluster 1 , reducing the frequency ratio of their excitatory inputs/inhibitory inputs, while counterbalancing this effect by favoring increased amplitudes of excitatory events relative to inhibitory ones. Effects on other clusters are more limited.

\section{Morphological Properties of CA1 Neurons from Cx30 deficient mice}

Somatic and dendritic morphologies of the neurons previously electrophysiologically characterized were successfully revealed for 32 out of the 44 recorded neurons following the biocytin labelling and Neurolucida reconstructions (Fig. 7a).

Regarding the somatic properties among the clusters identified in $\mathrm{C} \times 30$ deficient mice (data not shown), we did not observe statistical differences either between the clusters of the transgenic mice, or with respect to their corresponding clusters in the WT mice. The neurons of cluster 1 always had their somata localized in the pyramidal cell layer in both classifications. The cells of cluster 2 had their somata located either in the pyramidal cell layer or in the stratum oriens. Somata of cluster 3, 4 and 5 neurons were systematically located in stratum oriens. 


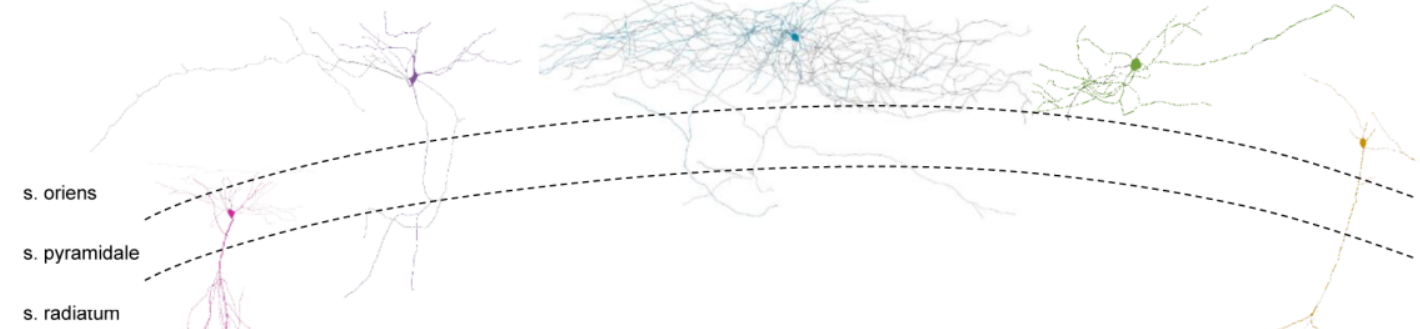

$\underline{100 \mu \mathrm{m}}$

b
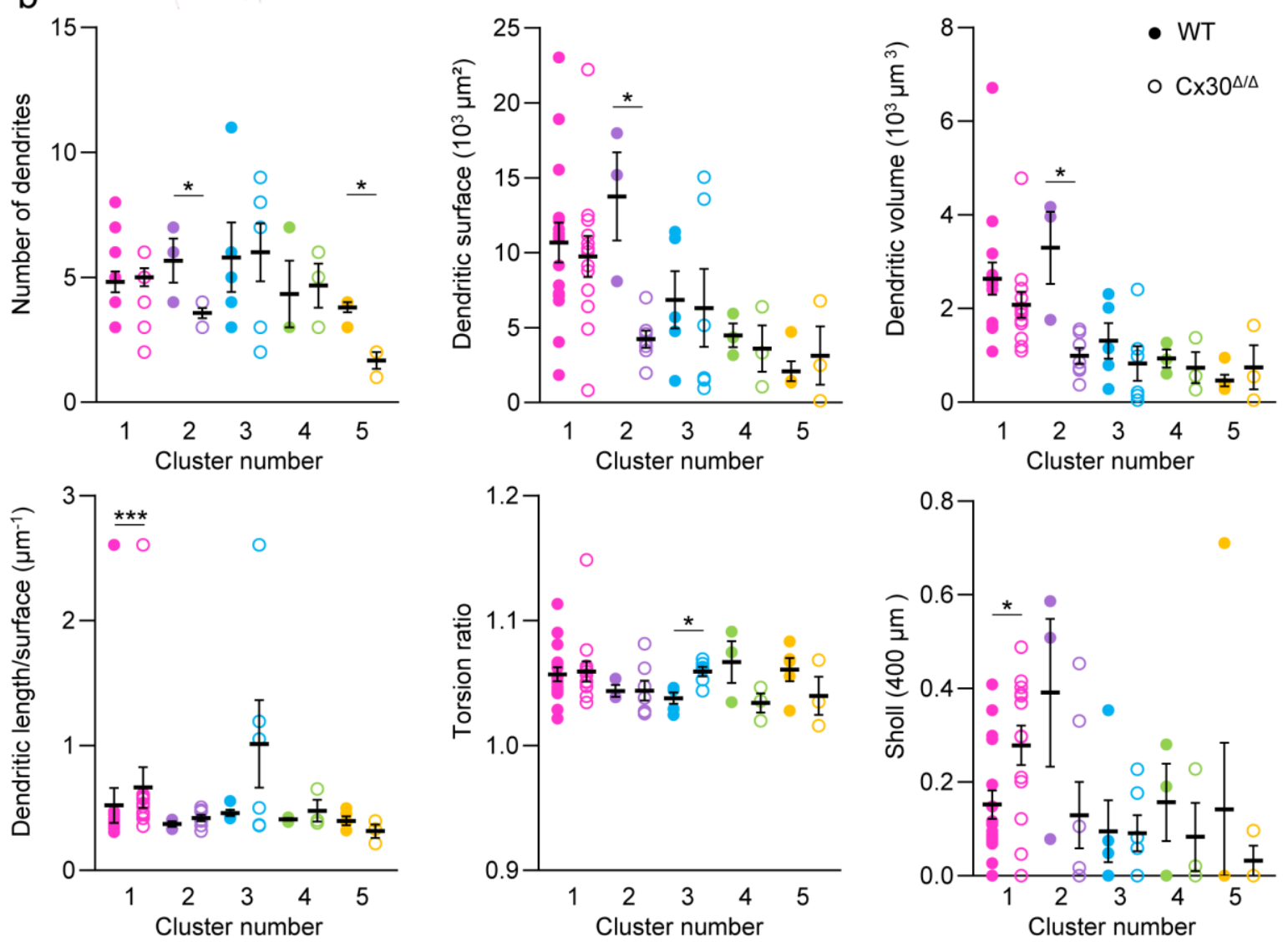

Figure 7. Morphological features of CA1 neurons in $\mathrm{Cx} 30^{\Delta / \Delta}$ mice. (a) Neurolucida reconstructions of typical neurons from each cluster. Axons are represented in gray and dendrites are color-coded according to their cluster. (b) Scatter dot plots representing the significant main morphological features of neurons in WT compared to $C \times 30^{\Delta / \Delta}$ mice, respectively to their belonging clusters. Mean values and SEM are represented by a black bar, Mann-Whitney U tests. 
However, numerous dendritic changes were observed among the clusters in Cx30 deficient mice compared to WT mice. In particular, neurons in cluster 2 and 5 from $\mathrm{C} \times 30^{\Delta / \Delta}$ mice were less ramified than in WT mice by $\sim 37 \%$ and $55 \%$ respectively (p39, Table 8; Fig. 7b). The dendritic surface and volume of neurons from cluster 2 were also decreased by $\sim 69 \%$ and $\sim 73 \%$ respectively in $C \times 30^{\Delta / \Delta}$ mice compared to WT mice (p42, Table 8; Fig. 7b). Neurons from $\mathrm{C} \times 30^{\Delta / \Delta}$ mice in cluster 1 had an increased dendritic length/surface ratio (43, Table 8$)$. We also found that the torsion ratio of cluster 3 neurons from $C \times 30^{\Delta / \Delta}$ mice was significantly increased by $\sim 10 \%$ compared to WT mice (p45, Table 8; Fig. 7b). Dendrites of neurons from cluster 1 of $C \times 30^{\Delta / \Delta}$ mice were longer, as their ramifications at $400 \mu \mathrm{m}$ were increased by $\sim 50 \%$ compared to WT mice (p50, Table 8), thereby increasing their dendritic length/surface ratio by $\sim 40 \%$ compared to WT mice (p43, Table 8, Fig. 7b). Overall, Cx30 seems to mainly modulate the dendritic features of pyramidal cells from cluster 1, extending their dendrites, as well as those of cluster 2, but in an opposite way, by decreasing their surface and volume, with limited effects on the other cell types. 
Table 8. Comparison of dendritic properties among CA1 neuron subtypes in $\mathrm{C} \times 30^{\Delta / \Delta}$ mice.

\begin{tabular}{|c|c|c|c|c|c|}
\hline & $\begin{array}{c}\text { Cluster } 1 \\
(n=13)\end{array}$ & $\begin{array}{c}\text { Cluster } 2 \\
(\mathrm{n}=7)\end{array}$ & $\begin{array}{c}\text { Cluster } 3 \\
(n=6)\end{array}$ & $\begin{array}{c}\text { Cluster } 4 \\
(n=3)\end{array}$ & $\begin{array}{c}\text { Cluster } \mathbf{5} \\
(\mathrm{n}=3)\end{array}$ \\
\hline (p39) $\mathbf{N b}$ of dendrites & $\begin{array}{c}5.0 \pm 0.4 \\
* 5\end{array}$ & $\begin{array}{c}3.6 \pm 0.2^{\star} \\
-\end{array}$ & $\begin{array}{c}6.0 \pm 1.1 \\
* 5\end{array}$ & $\begin{array}{c}4.7 \pm 0.9 \\
-\end{array}$ & $\begin{array}{c}1.7 \pm 0 . \mathbf{3}^{*} \\
* 1,3\end{array}$ \\
\hline$(p 40)$ Total length $\left(10^{3} \mu \mathrm{m}\right)$ & $\begin{array}{l}4.9 \pm 0.6 \\
* 2\end{array}$ & $\begin{array}{c}1.7 \pm 0.2^{*} \\
* 1\end{array}$ & $\begin{array}{c}5.3 \pm 2.4 \\
-\end{array}$ & $\begin{array}{c}1.7 \pm 0.6 \\
-\end{array}$ & $\begin{array}{c}1.1 \pm 0.6 \\
-\end{array}$ \\
\hline (p41) Surface $\left(10^{3} \mu \mathrm{m}^{2}\right)$ & $\begin{array}{c}9.7 \pm 1.4 \\
-\end{array}$ & $\begin{array}{c}4.2 \pm 0.6^{*} \\
-\end{array}$ & $\begin{array}{c}6.3 \pm 2.6 \\
-\end{array}$ & $\begin{array}{c}3.6 \pm 1.5 \\
-\end{array}$ & $\begin{array}{c}3.1 \pm 1.9 \\
-\end{array}$ \\
\hline (p42) Volume $\left(10^{3} \mu \mathrm{m}^{3}\right)$ & $\begin{array}{c}1.9 \pm 0.3 \\
-\end{array}$ & $\begin{array}{c}0.9 \pm 0.2^{*} \\
-\end{array}$ & $\begin{array}{c}0.8 \pm 0.4 \\
-\end{array}$ & $\begin{array}{c}0.7 \pm 0.3 \\
-\end{array}$ & $\begin{array}{c}0.7 \pm 0.4 \\
-\end{array}$ \\
\hline (p43) Length/surface $\left(\mu \mathrm{m}^{-1}\right)$ & $\begin{array}{c}0.7 \pm 0.2^{\star \star \star} \\
-\end{array}$ & $\begin{array}{c}0.4 \pm 0.0 \\
-\end{array}$ & $\begin{array}{c}1.0 \pm 0.4 \\
-\end{array}$ & $\begin{array}{c}0.5 \pm 0.1 \\
-\end{array}$ & $\begin{array}{c}0.3 \pm 0.1 \\
-\end{array}$ \\
\hline $\begin{array}{l}\text { (p44) Highest order } \\
\text { segment }\end{array}$ & $\begin{array}{c}17.9 \pm 2.6 \\
-\end{array}$ & $\begin{array}{c}5.9 \pm 0.7 \\
-\end{array}$ & $\begin{array}{c}10.3 \pm 2.7 \\
-\end{array}$ & $\begin{array}{c}4.6 \pm 0.3 \\
-\end{array}$ & $\begin{array}{c}4.7 \pm 1.9 \\
-\end{array}$ \\
\hline (p45) Torsion ratio & $\begin{array}{c}1.1 \pm 0.0 \\
-\end{array}$ & $\begin{array}{c}1.0 \pm 0.0 \\
-\end{array}$ & $\begin{array}{c}1.1 \pm 0.0^{*} \\
-\end{array}$ & $\begin{array}{c}1.0 \pm 0.0 \\
-\end{array}$ & $\begin{array}{c}1.0 \pm 0.0 \\
-\end{array}$ \\
\hline (p46) Nodes & $\begin{array}{c}41.5 \pm 5.6 \\
-\end{array}$ & $\begin{array}{c}12.7 \pm 2.4^{\star} \\
-\end{array}$ & $\begin{array}{c}38.3 \pm 18.2 \\
-\end{array}$ & $\begin{array}{c}9.7 \pm 1.9 \\
-\end{array}$ & $\begin{array}{c}6.7 \pm 3.4 \\
-\end{array}$ \\
\hline (p47) Sholl $100 \mu \mathrm{m}$ & $\begin{array}{c}0.3 \pm 0.0 \\
-\end{array}$ & $\begin{array}{c}0.4 \pm 0.1 \\
-\end{array}$ & $\begin{array}{c}0.5 \pm 0.1 \\
-\end{array}$ & $\begin{array}{c}0.6 \pm 0.2 \\
-\end{array}$ & $\begin{array}{c}0.7 \pm 0.2 \\
-\end{array}$ \\
\hline (p48) Sholl $200 \mu \mathrm{m}$ & $\begin{array}{c}0.3 \pm 0.0 \\
-\end{array}$ & $\begin{array}{c}0.3 \pm 0.0 \\
-\end{array}$ & $\begin{array}{c}0.2 \pm 0.0 \\
-\end{array}$ & $\begin{array}{c}0.2 \pm 0.1 \\
-\end{array}$ & $\begin{array}{c}0.2 \pm 0.1 \\
-\end{array}$ \\
\hline (p49) Sholl $300 \mu \mathrm{m}$ & $\begin{array}{c}0.1 \pm 0.0 \\
-\end{array}$ & $\begin{array}{c}0.1 \pm 0.0 \\
-\end{array}$ & $\begin{array}{c}0.1 \pm 0.0 \\
-\end{array}$ & $\begin{array}{c}0.1 \pm 0.1 \\
-\end{array}$ & $\begin{array}{c}0.1 \pm 0.1 \\
-\end{array}$ \\
\hline (p50) Sholl $400 \mu \mathrm{m}$ & $\begin{array}{c}0.3 \pm 0.0^{*} \\
-\end{array}$ & $\begin{array}{c}0.1 \pm 0.1 \\
-\end{array}$ & $\begin{array}{c}0.1 \pm 0.0 \\
-\end{array}$ & $\begin{array}{c}0.1 \pm 0.1 \\
-\end{array}$ & $\begin{array}{c}0.0 \pm 0.0 \\
-\end{array}$ \\
\hline
\end{tabular}

$n$, Number of cells; Kruskal-Wallis and Dunn's multiple comparisons test. Statistically different values from cluster in WT mice are written in bold, Mann-Whitney $U$-test. Asterisks indicate statistical significance $\left({ }^{*} P<0.05,{ }^{* * *} P<0.01\right)$ of the cluster in the column relative to the other clusters represented by their corresponding number. 


\section{Discussion}

We here investigated the role of astroglial $\mathrm{C} \times 30$ on the structural and functional properties of different subtypes of CA1 neurons. We found that astroglial Cx30 deficiency resulted in neurons generally endowed with a more depolarized action potential threshold and a higher saturation frequency of $\sim 22 \%$ with shorter AHP duration of $\sim 41 \%$. These results suggest that despite reduced neuronal excitability, these neurons can sustain a high discharge frequency. The impact of $\mathrm{C} \times 30$ deficiency is however not homogeneous among the different neuronal subtypes. This was also observed for mEPSC and mIPSCs and E/l balance, where pyramidal cells and interneurons were differentially impacted.

Five Neuronal Subpopulations Among CA1 Neurons from stratum oriens and pyramidale

In this study, we recorded 106 neurons from CA1 stratum oriens and pyramidale: 62 in WT and 44 in $C \times 30^{\Delta \Delta}$ mice. 28 electrophysiological parameters were extracted for each neuron. To investigate whether astroglial $\mathrm{C} \times 30$ deficiency alters neuronal properties, we first defined distinct neuronal subpopulations among CA1 neurons in WT mice, in order to compare the characteristics of each population to the ones of neurons from Cx30 deficient mice. We used the Ward's method to group cells with large similarities and to avoid personal bias of interpretation (Cauli et al., 1997).

In WT mice, we found that neurons segregated mainly in the cluster $1(46 \%)$. These neurons are characterized by a high membrane capacitance $\sim 89 \mathrm{pF}$, large action potential amplitude $\sim 93 \mathrm{mV}$, long action potential duration $\sim 1.5 \mathrm{~ms}$ with small AHP $\sim 5 \mathrm{mV}$, which are characteristic of pyramidal cells (Schwartzkroin, 1977). Spontaneous 
mEPSCs frequency is low $\sim 3 \mathrm{~Hz}$, as the amplitude of both mEPSC and mIPSCs, compared to neurons from others clusters. Their mEPSC/mIPSC ratio is high, with is in good agreement with the estimation that a typical CA1 pyramidal neuron receives about 30000 excitatory and 1700 inhibitory inputs (Megías et al., 2001). The morphology of the neurons in cluster 1 are also characteristic of pyramidal cells, with a very branched dendritic arborization in the stratum oriens and extending a single or two apical dendrites that reaches the border of stratum lacunosum moleculare, where it radiates into a number of thin tufted dendrites (Srinivas et al., 2017). The location of their soma, which is more superficial than the soma of neurons in other clusters, suggests that these cells correspond to superficial pyramidal cells (Cembrowski et al., 2016; Jarsky et al., 2008; Soltesz \& Losonczy, 2018).

In Cx30 $\Delta / \Delta$ mice, neurons from cluster 1 displayed a strong reduction in their AP duration, a key feature determining calcium entry in presynaptic terminals, and thus neurotransmitter release dynamics (Sabatini and Regehr, 1997, 1999). Moreover, the reduction of their AHP duration and the reduction of their action potential duration also favored an increased frequency of discharge (Fmax).

In $C \times 30 \Delta / \Delta$ mice, neurons from cluster 1 also displayed a decrease in the frequency of mEPSCs, as already described in pyramidal cells of another study using $\mathrm{C} \times 30^{-1-}$ deficient mice (Pannasch et al., 2014). Here, we further also observed an increase in the frequency of mIPSCs, drastically reducing the balance of mEPSCs/mIPSCs. No significant effect was observed regarding mIPSCs amplitude in WT compared to $\mathrm{C} \times 30^{\Delta / \Delta}$ mice, suggesting that $\mathrm{C} \times 30$ could regulate synaptic inputs, favoring the number of inhibitory terminals on cluster 1 neurons. Slight morphological alterations were found using Neurolucida reconstructions in neurons from $\mathrm{C} \times 30$ deficient mice, increasing the compactness or cell, e.g. the ramifications at $400 \mu \mathrm{m}$ and their ratio 
length/surface. Altogether, our observations in $\mathrm{C} \times 30^{\Delta / \Delta}$ mice may reflect a differential connectivity and functionality of cluster 1 pyramidal neurons.

Electrophysiologically, cluster 2 neurons were characterized by a low AHP amplitude of long duration $\sim 43 \mathrm{~ms}$, and by a pronounced accommodative hump. Regarding their Neurolucida reconstructions, we observed that these neurons also project to stratum laconosum-moleculare and may represent deeper pyramidal cells (Cembrowski et al., 2016; Jarsky et al., 2008; Soltesz \& Losonczy, 2018). In Cx30 deficient mice, cluster 2 neurons are the most affected electrophysiologically. Despite a more depolarized action potential threshold suggesting a decreased excitability, their membrane resistance, membrane time constant and their discharge at saturation is increased. The reduction of their AHP duration and the reduction of their action potential duration also favored an increased frequency of discharge (Fmax). Although not statistically significant, this strong reduction of their AHP duration, combined with the strong and significant increased amplitude of their AHP suggest an impact of $\mathrm{C} \times 30$ on ion channels responsible for AHP. These faster AHPs are then responsible for sustained high-frequency firing (Buhl et al., 1996).

The morphology of cluster 2 neurons was also significantly altered in Cx30 deficient mice. They appeared to be less developed, displaying a reduced number of dendrites, and thereby a reduced dendritic surface and volume. On the opposite to cluster 1 neurons, the frequency of their balance excitation over inhibition was enhanced in Cx30 deficient mice. The amplitude of mEPSCs/mIPSCs was also increased, similarly to cluster 1 and 5 neurons, probably triggering the enhanced firing frequency. 
Importantly, in contrast to AP of pyramidal cells, neurons from cluster 3 to 5 displayed action potentials of short duration, a characteristic of interneurons (Schwartzkroin \& Mathers, 1978).

Neurons from cluster 3 are characterized by a small membrane time constant and almost no sag ratio in wildtype mice. Unitary events recorded in these neurons had the highest mEPSCs frequency $(\sim 11 \mathrm{~Hz})$, suggesting a high excitatory synapse density, and high amplitudes of both mEPSCs and mIPSCs, plausibly from local pyramidal cells (Pawelzik et al., 2002). Morphologically, thesecells have a horizontal multipolar dendritic tree confined into stratum oriens and pyramidale. Their axonal arbors had strong projections to the stratum radiatum and to the subiculum, suggesting that these cells could correspond to trilaminar cells (Ferraguti, 2005; Pelkey et al., 2017) . In Cx30 deficient mice, their action potential threshold was again significantly enhanced and their sag ratio decreased. No effect on the excitatory-inhibitory balance was found. Regarding the Neurolucida reconstruction of cluster 3 neurons, the absence of Cx30 only increased the torsion ratio of their dendrites.

Neurons from cluster 4 displayed small action potential amplitudes and a very high discharge frequency at saturation $\sim 155 \mathrm{~Hz}$, which is the electrophysiological characteristic of fast-spiking interneurons (Hua Hu et al., 2014, 2018). Dendrites of this interneuron type were usually more vertically oriented with dendrites confined to the stratum oriens, as previously described (Booker et al., 2017; Lacaille et al., 1987; Pawelzik et al., 2002). Fast-spiking interneurons are typically parvalbumin (PV) expressing basket cells, but also include a subset of axo-axonic cells, bistratified cells (H. Hu et al., 2014; Szegedi et al., 2017). In Cx30 deficient mice, few changes were observed in this cluster. In particular, the frequency of unitary events recorded in these 
neurons increased by $52 \%$ (from 7.8 to $16.1 \mathrm{~Hz}$ ). Due to the small number of PV recorded neurons, we did not observe a significant change in the excitatory/inhibitory balance. However, in the absence of $\mathrm{Cx30}$, we observed a marked increase in the excitatory inputs, and in the opposite to all other clusters, their action potential threshold was not decreased, reflecting an hyperexcitability of PV neurons.

Finally, neurons from cluster 5 were electrophysiologically characterized by their very short first spike latency and their strong sag ratio, a typical feature of oriens lacunosum moleculare cells (O-LM) (Tricoire et al., 2011). Morphologically, these neurons display a small dendritic arborization and a long axon projecting from stratum oriens to lacunosum moleculare, confirming their identification as O-LM cells (Freund \& Buzsáki, 1996; Pelkey et al., 2017). Their length, surface and volume are small compared to the neurons from the other clusters. In $\mathrm{C} \times 30$ deficient mice, neurons from this cluster were the only ones to present a significant increase in their resting membrane potential and a reduced number of dendrites. As for all other types of neurons, the amplitude of their excitation/inhibitory balance was increased.

\section{Possible physiological mechanisms altering neuronal properties in Cx30 deficient mice}

Cx30 can interact with adjacent neurons to modulate neuronal network activities by multiple mechanisms involving both channel (gap-junction and hemichannel) and nonchannel functions ( Pannasch \& Rouach, 2013). Evidences from the literature reported that astrocytic coupling via GJ regulate neuronal synaptic and network activities (Chever et al., 2016; U. Pannasch et al., 2011; Ulrike Pannasch et al., 2012; Rouach et al., 2008; Wallraff et al., 2006). Beyond its role via GJ channels, Cx30 has also 
recently been shown to form hemichannels in hippocampal astrocytes (Ghézali et al., 2019). However, up to now physiological implications of $\mathrm{C} \times 30 \mathrm{HCs}$ have still not yet been reported, mostly due to the lack of specific tools to target them.

Interestingly, Cx30 can also interact with elements of the cytoskeleton (Qu et al., 2009), shown to regulate cell morphology, adhesion, and migration, independently of its channel functions. Remarkably, astroglial processes deficient for $\mathrm{C} \times 30$ have been reported to extend and invade hippocampal excitatory synapses, thereby regulating synaptic glutamate levels via clearance through channel-independent mechanism. Despite differences between $\mathrm{C} \times 30^{\Delta / \Delta}$ and $\mathrm{C} \times 30-/$ - mice (Boulay et al., 2013), we here speculate that a similar mechanism could also regulate astroglial morphology and synapses invasion in $C \times 30^{\Delta / \Delta}$ mice. Since astrocytes express GABA transporter 1 (GAT1) and 2 (GAT2) (Ghirardini et al., 2018), the vicinity of astroglial processes to inhibitory synapses could also directly regulate GABA clearance in the hippocampus (Juhász et al., 1997; Richards \& Bowery, 1996). Yet, GABA uptake by astrocytes was shown to be less important than glutamate uptake (Schousboe \& Waagepetersen, 2003). Differences between structural morphologies of excitatory and inhibitory synapses could also differentially allow astroglial invasion and neurotransmitter regulation. This could explain the differences observed regarding the regulation of the E/l balance throughout the different neuronal subtypes.

Importantly, we here observed few effects of Cx30 deficiency on fast-spiking neurons regulation, essentially only increasing the frequency of mEPSCs. This is in good agreement with previous observations, showing that the neuronal discharge of PV interneurons is not regulated by astrocytes (Tan et al., 2017). Moreover, it was shown by combining optogenetics and pharmacological approaches that astrocytes can detect synaptic activity of somatostatin expressing interneurons via $G A B A_{B} R$ and GAT- 
3-dependent calcium signaling mechanisms, but not PV expressing interneurons inhibition, uncovering distinct functional interactions between specific subpopulations of interneurons and astrocytes (Matos et al., 2018) .

In epileptic conditions, a high regime of activity, deletion of $\mathrm{C} \times 30$ in $\mathrm{C} \times 30-/-$ mice reduced bursting activity (Pannasch et al., 2019). Here we show that in $C \times 30^{\Delta / \Delta}$ mice, Cx30 deficiency sustains high firing frequencies at saturation. However, the regimes at play in both studies are very different. In epileptic condition, neuronal network excitation is increased, whereas in our study, we only depolarized one cell, avoiding the saturation of neurotransmitters buffering by astroglial processes. It is thus difficult to directly compare these studies. Interestingly, HCs being activated by sustained neuronal activity (Chever et al., 2014; Pannasch et al., 2014), they could also contribute to regulate the activity in epileptic network bursting.

Regarding all these results, we can speculate that in our study, channel independent functions of Cx30 may regulate basal synaptic activity, likely via differential modulation astroglial glutamate and GABA transport.

In all, we here identified a role for astroglial $\mathrm{C} \times 30$ on neuronal properties and both excitatory and inhibitory inputs to distinct neuronal subtypes. These results provide new perspectives on the way astrocytes contribute, as non-neuronal components, to neuronal network functioning.

\section{Conflict of interest}

The authors declare no conflict of interest. 


\section{Data Availability}

The data that support the findings of this study are available from the corresponding author upon reasonable request.

\section{Acknowledgments and Fundings}

This work was supported by the Centre National de la Recherche Scientifique (CNRS), the French Institute of Health and Medical Research (Inserm), and the Collège de France. This work was also supported by grants from the European Research Council (Consolidator grant \#683154) and European Union's Horizon 2020 research and innovation program (Marie Sklodowska-Curie Innovative Training Networks, grant \#722053, EU-GliaPhD) to N.R. We thank all members of the animal house facility from the Collège de France. We are grateful to Quentin Perrenoud, for his help in Matlab programming for $\mathrm{K}$ mean corrections of cluster analysis.

This is the pre-peer reviewed version of our article, which has been published in final form at DOI: 10.1002/glia.24017. This article may be used for non-commercial purposes in accordance with Wiley Terms and Conditions for Use of Self-Archived Versions. 


\section{References}

Adesnik, H. (2018). Layer-specific excitation/inhibition balances during neuronal synchronization in the visual cortex. The Journal of Physiology, 596(9), 16391657. doi : $10.1113 / J P 274986$

Araque, A., Carmignoto, G., Haydon, P. G., Oliet, S. H. R., Robitaille, R., \& Volterra, A. (2014). Gliotransmitters travel in time and space. Neuron, 81(4), 728-739. doi : 10.1016/j.neuron.2014.02.007

Ascoli, G. A., Alonso-Nanclares, L., Anderson, S. A., Barrionuevo, G., BenavidesPiccione, R., Burkhalter, A., Buzsáki, G., Cauli, B., DeFelipe, J., Fairén, A., Feldmeyer, D., Fishell, G., Fregnac, Y., Freund, T. F., Gardner, D., Gardner, E. P., Goldberg, J. H., Helmstaedter, M., Hestrin, S., .. Yuste, R. (2008). Petilla terminology: nomenclature of features of GABAergic interneurons of the cerebral cortex. Nature Reviews Neuroscience, 9(7), 557-568. doi : 10.1038/nrn2402

Bhatia, A., Moza, S., \& Bhalla, U. S. (2019). Precise excitation-inhibition balance controls gain and timing in the hippocampus. ELife, 8. doi : 10.7554/eLife.43415

Booker, S. A., Althof, D., Degro, C. E., Watanabe, M., Kulik, Á., \& Vida, I. (2017). Differential surface density and modulatory effects of presynaptic GABAB receptors in hippocampal cholecystokinin and parvalbumin basket cells. Brain Structure and Function, 222(8), 3677-3690. doi : 10.1007/s00429-017-1427-x

Boulay, A. C., del Castillo, F. J., Giraudet, F., Hamard, G., Giaume, C., Petit, C., Avan, P., \& Cohen-Salmon, M. (2013). Hearing is normal without connexin30. Journal of Neuroscience, 33(2), 430-434. doi : 10.1523/JNEUROSCI.4240-12.2013

Buhl, E. H., Szilágyi, T., Halasy, K., \& Somogyi, P. (1996). Physiological properties of anatomically identified basket and bistratified cells in the CA1 area of the rat hippocampus in vitro. Hippocampus, 6(3), 294-305. doi : 10.1002/(SICI)10981063(1996)6:3<294::AID-HIPO7>3.0.CO;2-N

Cauli, B., Audinat, E., Lambolez, B., Angulo, M. C., Ropert, N., Tsuzuki, K., Hestrin, S., \& Rossier, J. (1997). Molecular and physiological diversity of cortical nonpyramidal cells. The Journal of Neuroscience: The Official Journal of the Society for Neuroscience, 17(10), 3894-3906. doi : 10.1523/JNEUROSCI.17-1003894.1997 
Cembrowski, M. S., Bachman, J. L., Wang, L., Sugino, K., Shields, B. C., \& Spruston, N. (2016). Spatial Gene-Expression Gradients Underlie Prominent Heterogeneity of CA1 Pyramidal Neurons. Neuron, 89(2), 351-368. doi : 10.1016/j.neuron.2015.12.013

Chever, O., Dossi, E., Pannasch, U., Derangeon, M., \& Rouach, N. (2016). Astroglial networks promote neuronal coordination. Science Signaling, 9(410), 1-9. doi : 10.1126/scisignal.aad3066

Chever, O., Lee, C. Y., \& Rouach, N. (2014). Astroglial connexin43 hemichannels tune basal excitatory synaptic transmission. Journal of Neuroscience, 34(34), 1122811232. doi : 10.1523/JNEUROSCI.0015-14.2014

Covelo, A., \& Araque, A. (2018). Neuronal activity determines distinct gliotransmitter release from a single astrocyte. ELife, 7. doi : 10.7554/eLife.32237

Eroglu, C., \& Barres, B. A. (2010). Regulation of synaptic connectivity by glia. In Nature (Vol. 468, Issue 7321, pp. 223-231). Nature. doi : 10.1038/nature09612

Ferguson, B. R., \& Gao, W.-J. (2018). PV Interneurons: Critical Regulators of E/I Balance for Prefrontal Cortex-Dependent Behavior and Psychiatric Disorders. Frontiers in Neural Circuits, 12. doi : 10.3389/fncir.2018.00037

Ferraguti, F. (2005). Metabotropic Glutamate Receptor 8-Expressing Nerve Terminals

Target Subsets of GABAergic Neurons in the Hippocampus. Journal of Neuroscience, 25(45), 10520-10536. doi : 10.1523/JNEUROSCI.2547-05.2005

Freund, T. F., \& Buzsáki, G. (1996). Interneurons of the hippocampus. Hippocampus, 6(4), 347-470. doi : 10.1002/(SICI)1098-1063(1996)6:4<347::AIDHIPO1>3.0.CO;2-I.

Genoud, C., Quairiaux, C., Steiner, P., Hirling, H., Welker, E., \& Knott, G. W. (2006). Plasticity of Astrocytic Coverage and Glutamate Transporter Expression in Adult Mouse Cortex. PLoS Biology, 4(11), e343. doi : 10.1371/journal.pbio.0040343

Ghézali, G., Dallérac, G., \& Rouach, N. (2016). Perisynaptic astroglial processes: dynamic processors of neuronal information. Brain Structure and Function, 221(5), 2427-2442. doi : 10.1007/s00429-015-1070-3

Ghézali, G., Vasile, F., Curry, N., Fantham, M., Cheung, G., Ezan, P., Cohen-Salmon, 
M., Kaminski, C., \& Rouach, N. (2019). Neuronal Activity Drives Astroglial Connexin 30 in Perisynaptic Processes and Shapes Its Functions. Cerebral Cortex. doi : 10.1093/cercor/bhz123

Ghirardini, E., Wadle, S. L., Augustin, V., Becker, J., Brill, S., Hammerich, J., Seifert, G., \& Stephan, J. (2018). Expression of functional inhibitory neurotransmitter transporters GlyT1, GAT-1, and GAT-3 by astrocytes of inferior colliculus and hippocampus. Molecular Brain, 11(1), 4. doi : 10.1186/s13041-018-0346-y

Hansen, D. B., Ye, Z. C., Calloe, K., Braunstein, T. H., Hofgaard, J. P., Ransom, B. R., Nielsen, M. S., \& MacAulay, N. (2014). Activation, permeability, and inhibition of astrocytic and neuronal large pore (Hemi)channels. Journal of Biological Chemistry, 289(38), 26058-26073. doi : 10.1074/jbc.M114.582155

Hu, H., Gan, J., \& Jonas, P. (2014). Fast-spiking, parvalbumin+ GABAergic interneurons: From cellular design to microcircuit function. Science, 345(6196), 1255263-1255263. doi : 10.1126/science.1255263

Hu, Hua, Gan, J., \& Jonas, P. (2014). Interneurons. Fast-spiking, parvalbumin+ GABAergic interneurons: from cellular design to microcircuit function. Science (New York, N.Y.), 345(6196), 1255263. doi : 10.1126/science.1255263

Hu, Hua, Roth, F. C., Vandael, D., \& Jonas, P. (2018). Complementary Tuning of Na+ and $\mathrm{K}+$ Channel Gating Underlies Fast and Energy-Efficient Action Potentials in GABAergic Interneuron Axons. Neuron, 98(1), 156-165.e6. doi : 10.1016/j.neuron.2018.02.024

lascone, D. M., Li, Y., Sümbül, U., Doron, M., Chen, H., Andreu, V., Goudy, F., Blockus, H., Abbott, L. F., Segev, I., Peng, H., \& Polleux, F. (2020). Whole-Neuron Synaptic Mapping Reveals Spatially Precise Excitatory/Inhibitory Balance Limiting Dendritic and Somatic Spiking. Neuron. doi : 10.1016/j.neuron.2020.02.015

Jarsky, T., Mady, R., Kennedy, B., \& Spruston, N. (2008). Distribution of bursting neurons in the CA1 region and the subiculum of the rat hippocampus. Journal of Comparative Neurology, 506(4), 535-547. doi : 10.1002/cne.21564

Juhász, G., Kékesi, K. A., Nyitrai, G., Dobolyi, A., Krogsgaard-Larsen, P., \& Schousboe, A. (1997). Differential effects of nipecotic acid and 4,5,6,7tetrahydroisoxazolo[4,5-c]pyridin-3-ol on extracellular $\alpha$-aminobutyrate levels in 
rat thalamus. European Journal of Pharmacology, 331(2-3), 139-144. doi : 10.1016/S0014-2999(97)01044-3

Kanichay, R. T., \& Silver, R. A. (2008). Synaptic and cellular properties of the feedforward inhibitory circuit within the input layer of the cerebellar cortex. Journal of Neuroscience, 28(36), 8955-8967. doi : 10.1523/JNEUROSCI.5469-07.2008

Karagiannis, A., Gallopin, T., Dávid, C., Battaglia, D., Geoffroy, H., Rossier, J., Hillman, E. M. C., \& Staiger, J. F. (2009). Classification of NPY-expressing neocortical interneurons. The Journal of Neuroscience, 29(11), 3642-3659. doi : 10.1523/JNEUROSCI.0058-09.2009.Classification

Klausberger, T., \& Somogyi, P. (2008). Neuronal diversity and temporal dynamics: The unity of hippocampal circuit operations. In Science (Vol. 321, Issue 5885, pp. 5357). Science. doi : 10.1126/science.1149381

Lacaille, J. C., Mueller, A. L., Kunkel, D. D., \& Schwartzkroin, P. A. (1987). Local circuit interactions between oriens/alveus interneurons and CA1 pyramidal cells in hippocampal slices: Electrophysiology and morphology. Journal of Neuroscience, 7(7), 1979-1993. doi : 10.1523/jneurosci.07-07-01979.1987

Lee, E., Lee, J., \& Kim, E. (2017). Excitation/Inhibition Imbalance in Animal Models of Autism Spectrum Disorders. Biological Psychiatry, 81(10), 838-847. doi : 10.1016/j.biopsych.2016.05.011

Matos, M., Bosson, A., Riebe, I., Reynell, C., Vallée, J., Laplante, I., Panatier, A., Robitaille, R., \& Lacaille, J. C. (2018). Astrocytes detect and upregulate transmission at inhibitory synapses of somatostatin interneurons onto pyramidal cells. Nature Communications, 9(1). doi : 10.1038/s41467-018-06731-y

Megías, M., Emri, Z., Freund, T. ., \& Gulyás, A. . (2001). Total number and distribution of inhibitory and excitatory synapses on hippocampal CA1 pyramidal cells. Neuroscience, 102(3), 527-540. doi : 10.1016/S0306-4522(00)00496-6

Murphy, B. K., \& Miller, K. D. (2009). Balanced Amplification: A New Mechanism of Selective Amplification of Neural Activity Patterns. Neuron, 61(4), 635-648. doi : 10.1016/j.neuron.2009.02.005

Nelson, S. B., \& Valakh, V. (2015). Excitatory/Inhibitory Balance and Circuit Homeostasis in Autism Spectrum Disorders. Neuron, 87(4), 684-698. doi : 
10.1016/j.neuron.2015.07.033

Okun, M., \& Lampl, I. (2008). Instantaneous correlation of excitation and inhibition during ongoing and sensory-evoked activities. Nature Neuroscience, 11(5), 535537. doi : $10.1038 / \mathrm{nn} .2105$

Oliet, S. H., Piet, R., \& Poulain, D. A. (2001). Control of glutamate clearance and synaptic efficacy by glial coverage of neurons. Science (New York, N.Y.), 292(5518), 923-926. doi : 10.1126/science.1059162

Pannasch, U., Vargova, L., Reingruber, J., Ezan, P., Holcman, D., Giaume, C., Sykova, E., \& Rouach, N. (2011). Astroglial networks scale synaptic activity and plasticity. Proceedings of the National Academy of Sciences, 108(20), 8467-8472. doi : 10.1073/pnas.1016650108

Pannasch, U, \& Rouach, N. (2013). Emerging role for astroglial networks in information processing: From synapse to behavior. In Trends in Neurosciences (Vol. 36, Issue 7, pp. 405-417). doi : 10.1016/j.tins.2013.04.004

Pannasch, Ulrike, Derangeon, M., Chever, O., \& Rouach, N. (2012). Astroglial gap junctions shape neuronal network activity. Communicative \& Integrative Biology, 5(3), 248-254. doi : 10.4161/cam.20753

Pannasch, Ulrike, Dossi, E., Ezan, P., \& Rouach, N. (2019). Astroglial Cx30 sustains neuronal population bursts independently of gap-junction mediated biochemical coupling. Glia, 67(6), 1104-1112. doi : 10.1002/glia.23591

Pannasch, Ulrike, Freche, D., Dallérac, G., Ghézali, G., Escartin, C., Ezan, P., CohenSalmon, M., Benchenane, K., Abudara, V., Dufour, A., Lübke, J. H. R., Déglon, N., Knott, G., Holcman, D., \& Rouach, N. (2014). Connexin 30 sets synaptic strength by controlling astroglial synapse invasion. Nature Neuroscience, 17(4), 549-558. doi : 10.1038/nn.3662

Pawelzik, H., Hughes, D. I., \& Thomson, A. M. (2002). Physiological and morphological diversity of immunocytochemically defined parvalbumin- and cholecystokininpositive interneurones in CA1 of the adult rat hippocampus. Journal of Comparative Neurology, 443(4), 346-367. doi : 10.1002/cne.10118

Pelkey, K. A., Chittajallu, R., Craig, M. T., Tricoire, L., Wester, J. C., \& McBain, C. J. (2017). Hippocampal GABAergic Inhibitory Interneurons. Physiological Reviews, 
97(4), 1619-1747. doi : 10.1152/physrev.00007.2017

Perrenoud, Q., Geoffroy, H., Gauthier, B., Rancillac, A., Alfonsi, F., Kessaris, N., Rossier, J., Vitalis, T., \& Gallopin, T. (2012). Characterization of Type I and Type II nNOS-Expressing Interneurons in the Barrel Cortex of Mouse. Frontiers in Neural Circuits, 6(June), 1-17. doi : 10.3389/fncir.2012.00036

Qu, C., Gardner, P., \& Schrijver, I. (2009). The role of the cytoskeleton in the formation of gap junctions by Connexin 30. Experimental Cell Research, 315(10), 16831692. doi : 10.1016/j.yexcr.2009.03.001

Richards, D. A., \& Bowery, N. G. (1996). Comparative effects of the GABA uptake inhibitors, tiagabine and NNC-711, on extracellular GABA levels in the rat ventrolateral thalamus. Neurochemical Research, 21(2), 135-140. doi : 10.1007/BF02529130

Rouach, N., Koulakoff, A., Abudara, V., Willecke, K., \& Giaume, C. (2008). Astroglial Metabolic Networks Sustain Hippocampal Synaptic Transmission. Science (New York, N.Y.), 322(December), 1551-1555. doi : 10.1126/science.1164022

Roux, L., \& Buzsáki, G. (2015). Tasks for inhibitory interneurons in intact brain circuits. Neuropharmacology, 88, 10-23. doi : 10.1016/j.neuropharm.2014.09.011

Rusakov, D. A. (2015). Disentangling calcium-driven astrocyte physiology. Nature Reviews. Neuroscience, 16(4), 226-233. doi : 10.1038/nrn3878

Sangare, A., Dubourget, R., Geoffroy, H., Gallopin, T., \& Rancillac, A. (2016). Serotonin differentially modulates excitatory and inhibitory synaptic inputs to putative sleep-promoting neurons of the ventrolateral preoptic nucleus. Neuropharmacology, 109, 29-40. doi : 10.1016/j.neuropharm.2016.05.015

Schousboe, A., \& Waagepetersen, H. S. (2003). Role of astrocytes in homeostasis of glutamate and GABA during physiological and pathophysiological conditions. Advances in Molecular and Cell Biology, 31, 461-474. doi : 10.1016/S15692558(03)31020-3

Schwartzkroin, P. A. (1977). Further characteristics of hippocampal CA1 cells in vitro. Brain Research, 128(1), 53-68. doi : 10.1016/0006-8993(77)90235-9

Schwartzkroin, P. A., \& Mathers, L. H. (1978). Physiological and morphological 
identification of a nonpyramidal hippocampal cell type. Brain Research, 157(1), 110. doi : 10.1016/0006-8993(78)90991-5

Sholl, D. A. (1953). Dendritic organization in the neurons of the visual and motor cortices of the cat. J Anat., 87(4), 387-406.

Soltesz, I., \& Losonczy, A. (2018). CA1 pyramidal cell diversity enabling parallel information processing in the hippocampus. Nature Neuroscience, 21(4), 484493. doi : 10.1038/s41593-018-0118-0

Srinivas, K. V., Buss, E. W., Sun, Q., Santoro, B., Takahashi, H., Nicholson, D. A., \& Siegelbaum, S. A. (2017). The Dendrites of CA2 and CA1 Pyramidal Neurons Differentially Regulate Information Flow in the Cortico-Hippocampal Circuit. The Journal of Neuroscience, 37(12), 3276-3293. doi : 10.1523/JNEUROSCI.221916.2017

Staff, N. P., Jung, H.-Y., Thiagarajan, T., Yao, M., \& Spruston, N. (2000). Resting and Active Properties of Pyramidal Neurons in Subiculum and CA1 of Rat Hippocampus. Journal of Neurophysiology, 84(5), 2398-2408. doi : 10.1152/jn.2000.84.5.2398

Szegedi, V., Molnár, G., Paizs, M., Csakvari, E., Barzó, P., Tamás, G., \& Lamsa, K. (2017). High-Precision Fast-Spiking Basket Cell Discharges during Complex Events in the Human Neocortex. ENeuro, 4(5). doi : 10.1523/ENEURO.026017.2017

Tan, Z., Liu, Y., Xi, W., Lou, H., Zhu, L., Guo, Z., Mei, L., \& Duan, S. (2017). Gliaderived ATP inversely regulates excitability of pyramidal and CCK-positive neurons. Nature Communications, 8, 13772. doi : 10.1038/ncomms 13772

Tricoire, L., Pelkey, K. A., Erkkila, B. E., Jeffries, B. W., Yuan, X., \& McBain, C. J. (2011). A blueprint for the spatiotemporal origins of mouse hippocampal interneuron diversity. Journal of Neuroscience, 31(30), 10948-10970. doi : 10.1523/JNEUROSCI.0323-11.2011

Wallraff, A., Köhling, R., Heinemann, U., Theis, M., Willecke, K., \& Steinhäuser, C. (2006). The impact of astrocytic gap junctional coupling on potassium buffering in the hippocampus. Journal of Neuroscience, 26(20), 5438-5447. doi : 10.1523/JNEUROSCI.0037-06.2006 
Ward, J. H. (1963). Hierarchical Grouping to Optimize an Objective Function. Journal of the American Statistical Association, 58(301), 236. doi : 10.2307/2282967

Wehr, M., \& Zador, A. M. (2003). Balanced inhibition underlies tuning and sharpens spike timing in auditory cortex. Nature, 426(6965), 442-446. doi : 10.1038/nature02116

Yizhar, O., Fenno, L. E., Prigge, M., Schneider, F., Davidson, T. J., O’Shea, D. J., Sohal, V. S., Goshen, I., Finkelstein, J., Paz, J. T., Stehfest, K., Fudim, R., Ramakrishnan, C., Huguenard, J. R., Hegemann, P., \& Deisseroth, K. (2011). Neocortical excitation/inhibition balance in information processing and social dysfunction. Nature, 477(7363), 171-178. doi : 10.1038/nature10360 


\section{Supplementary Figure and Legend}
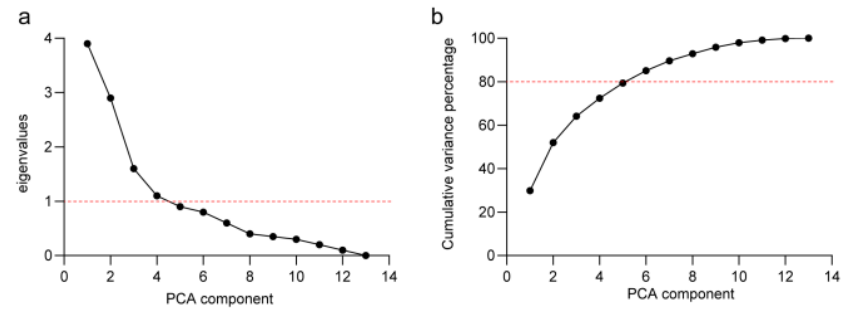

Supplementary Figure S1. Principal component analysis. (a) Scree plot of eigenvalues and PCA component. The red dot line represents the Kaiser criterion value for eigenvalues $>1$. (b) Cumulative variance percentage for PCA component. The red dot line represents the $80 \%$ of variance representation. 